DR. MELINDA FITZGERALD (Orcid ID : 0000-0002-4823-8179)

Article type : Original Article

\title{
Differential responses to increasing numbers of mild traumatic brain injury in a rodent
}

\section{closed head injury model}

Brooke Fehily ${ }^{1,2}$, Carole A. Bartlett ${ }^{1}$, Stephen Lydiard ${ }^{1}$, Michael Archer $^{1}$, Hannah Milbourn ${ }^{1}$, Maimuna Majimbi $^{1,3}$, Jan M. Hemmi ${ }^{1}$, Sarah, A. Dunlop ${ }^{1}$, Nathanael J. Yates ${ }^{2}$ and Melinda Fitzgerald ${ }^{1,3,4}$

${ }^{1}$ Experimental and Regenerative Neurosciences, School of Biological Sciences, ${ }^{2}$ School of Human Sciences, The University of Western Australia, 35 Stirling Hwy, Nedlands, 6009 Western Australia, Australia, ${ }^{3}$ Curtin Health Innovation Research Institute, Faculty of Health Sciences, ${ }^{4}$ The Perron Institute for Neurological and Translational Science, Sarich Neuroscience Research Institute Building, 8 Verdun St, Nedlands, 6009 Western Australia, Australia

\section{Running Title:}

Responses to increasing numbers of $\mathrm{mTBI}$

Keywords: repeated mild traumatic brain injury; functional deficits; oxidative stress; myelin abnormalities; microglia; transmission electron microscopy

This article has been accepted for publication and undergone full peer review but has not been through the copyediting, typesetting, pagination and proofreading process, which may lead to differences between this version and the Version of Record. Please cite this article as doi: $10.1111 /$ jnc. 14673

This article is protected by copyright. All rights reserved. 


\section{Corresponding author:}

Melinda Fitzgerald, Prof, PhD

Curtin Health Innovation Research Institute, Curtin University and the Perron Institute. Sarich Neuroscience Research Institute Building 8 Verdun Street, Nedlands, WA, 6009, Australia

Tel +61 86488 2353, Fax +61 86488 1029, Email lindy.fitzgerald@curtin.edu.au

\section{Contributing authors:}

Brooke Fehily, BSc (Hons)

School of Human Sciences, The University of Western Australia (M309)

35 Stirling Highway, Crawley, WA, 6009, Australia

Tel +61 86488 3524, Fax +61 86488 1029, Email brooke.fehily@uwa.edu.au

Carole A. Bartlett, MSc

School of Biological Sciences, The University of Western Australia (M317)

35 Stirling Highway, Crawley, WA, 6009, Australia

Tel +61 86488 3524, Fax +61 86488 1029, Email carole.bartlett@uwa.edu.au

\section{Stephen Lydiard}

School of Biological Sciences, The University of Western Australia (M317)

35 Stirling Highway, Crawley, WA, 6009, Australia

Tel +61 86488 3524, Fax +61 86488 1029, Email stephen.lydiard@bath.edu

\section{Michael Archer}

School of Biological Sciences, The University of Western Australia (M317)

35 Stirling Highway, Crawley, WA, 6009, Australia

Tel +61 86488 3524, Fax +61 86488 1029, Email michael.archer@uwa.edu.au

This article is protected by copyright. All rights reserved. 
Hannah Milbourn

c/- School of Biological Sciences, The University of Western Australia (M317)

35 Stirling Highway, Crawley, WA, 6009, Australia

Tel +61 86488 3524, Fax+61 86488 1029, Email hm525@bath.ac.uk

Maimuna Majimbi

Curtin Health Innovation Research Institute, Curtin University

Sarich Neuroscience Research Institute

8 Verdun Street, Nedlands, WA, 6009, Australia

Tel +61 86488 2353, Fax +61 86488 1029, Email maimuna.majimbi@postgrad.curtin.edu.au

Jan M. Hemmi, PhD

School of Biological Sciences, The University of Western Australia (M092)

35 Stirling Highway, Crawley, WA, 6009, Australia

Tel +61 86488 3117, Fax +61 86488 7527, Email jan.hemmi@uwa.edu.au

Sarah A. Dunlop, Prof, PhD

School of Biological Sciences, The University of Western Australia (M092)

35 Stirling Highway, Crawley, WA, 6009, Australia

Tel +61 86488 2237, Fax +61 86488 7527, Email sarah.dunlop@uwa.edu.au

Nathanael J. Yates, PhD

School of Human Sciences, The University of Western Australia (M317)

35 Stirling Highway, Crawley, WA, 6009, Australia

Tel +61 86488 3489, Email nathanael.yates@uwa.edu.au

This article is protected by copyright. All rights reserved. 


\section{List of Abbreviations}

ANOVA Analysis of variance

Cat\# catalogue number

DAB 3,3'-diaminobenzadine

GFAP glial fibrillary acidic protein

iNOS inducible nitric oxide synthase

LW ladder walk

mTBI mild traumatic brain injury

MWM Morris water maze

PBS phosphate buffered saline

RRID Research Resource Identifiers

TEM transmission electron microscopy

8OHdG 8-hydroxy-2'-deoxyguanosine

\section{Abstract}

Following mild traumatic brain injury (mTBI), further mild impacts can exacerbate negative outcomes. To compare chronic damage and deficits following increasing numbers of repeated mTBIs, a closed-head weight-drop model of repeated mTBI was used to deliver 1,2 or 3 mTBIs to adult female rats at $24 \mathrm{~h}$ intervals. Outcomes were assessed at 3 months following the first mTBI. No gross motor, sensory or reflex deficits were identified $(p>0.05)$, consistent with current literature. Cognitive function assessed using a Morris water maze revealed chronic memory deficits following 1 and 2 , but not $3 \mathrm{mTBI}$ compared to shams $(\mathrm{p} \leq 0.05)$. Oxidative damage to DNA was assessed immunohistochemically in the dentate hilus of the hippocampus and splenium of the corpus

This article is protected by copyright. All rights reserved. 
callosum; no changes were observed. IBA1 positive microglia were increased in size in the cortex following $1 \mathrm{mTBI}$ and in the corpus callosum following 2 mTBI compared to shams ( $p \leq 0.05$ ); no changes were observed in the dentate hilus. GFAP positive astrocyte immunoreactivity was assessed in all three brain regions and no chronic changes were observed. Integrity of myelin ultrastructure in the corpus callosum was assessed using transmission electron microscopy. $\mathrm{G}$ ratio was decreased following 2 mTBIs compared to shams $(p \leq 0.05)$ at post-hoc level only. The changing patterns of damage and deficits following increasing numbers of $\mathrm{mTBI}$ may reflect dynamic responses to small numbers of mTBls or a conditioning effect such that increasing numbers of mild traumatic brain injuries do not necessarily result in worsening pathology.

\section{Introduction}

Epidemiological research estimates that $70-90 \%$ of all TBIs are mild (Cassidy et al. 2004, Government 2011) and therefore mTBls represent a significant public healthcare concern. Symptoms are generally transient, however can persist in some patients, who may experience longterm cognitive dysfunction (Carroll et al. 2014, Rivara \& Graham 2014, Willer \& Leddy 2006). Additional mild impacts are common in sport and military settings and can result in detrimental effects such as mild cognitive impairment that persist and worsen with time (McCrea et al. 2015, Martini \& Broglio 2017). However, there is considerable variability between individuals in terms of whether 2, 3 or more mTBIs result in long term cognitive impairment (McCrory et al. 2017). Negative outcomes are thought to be worse for females (Shahrokhi et al. 2012, Maher et al. 2014) or when repeated impacts occur close together in time, within a 'window of cerebral vulnerability' (Blennow et al. 2016). When managing $\mathrm{mTBI}$, clinicians are faced with a lack of knowledge regarding the likelihood of long-term harm, particularly when patients experience further insults. How many

This article is protected by copyright. All rights reserved. 
concussions are "too many", how "severe" does the concussion have to be, and at what point is action warranted to diminish risk, such as retirement from sport?

To answer questions critical to understanding the pathology of impact-induced repeated mTBI, it is necessary to utilise a model that introduces both linear and rotational acceleration as well as deceleration forces to the brain, resulting in diffuse rather than focal damage (Holbourn 1943, Dashnaw et al. 2012, Meaney \& Smith 2011). Studies of repeated mTBI have typically been conducted using weight drop closed head injuries in male mice, with some incorporating rotation and therefore generating a diffuse injury reminiscent of clinical mTBI (Fehily \& Fitzgerald 2016). Study designs have encompassed various comparisons between 1-30 mTBI (Donovan et al. 2014, Huang et al. 2013, Xu et al. 2016, Shitaka et al. 2011, Briggs et al. 2016, McAteer et al. 2016, Shultz et al. 2012, Mountney et al. 2017) (selected references shown), particularly focusing on cognitive measures, basic histology, traumatic axonal injury and inflammation. Acute learning, memory and/ or motor deficits have been reported to persist to both sub-acute (Shitaka et al. 2011, Namjoshi et al. 2014, Yang et al. 2015) and chronic time points (Hylin et al. 2013, Meehan et al. 2012, Luo et al. 2014, Thomsen et al. 2016), while longer inter-injury intervals dampen deficits and appear to confer protection against neuroinflammatory and axonal pathologies (Weil et al. 2014, Longhi et al. 2005, Meehan et al. 2012, Mannix et al. 2013). The majority of rodent studies use either 1 or 2 day interinjury intervals or less (Meehan et al. 2012, Mannix et al. 2013, Nichols et al. 2016, Bolton Hall et al. 2016), and it has been demonstrated that a window of cerebral vulnerability exists, during which an additional injury can worsen outcomes (Prins et al. 2013, Selwyn et al. 2016). However, studies to date have not utilised experimental paradigms designed to analyse chronic mechanisms of pathology comparing differences following 1,2 , or 3 mTBI. Such paradigms might allow an understanding of long-term responses that change with each additional injury and assessing chronic outcomes following a small number of injuries is necessary to inform clinical decisions and 'return to play' periods in sports.

This article is protected by copyright. All rights reserved. 
Cell-specific elements of pathology following mTBI include changes to glia that are likely to impact upon function. Astrocyte hypertrophy and microglial activation are well described phenomena following traumatic injury to the central nervous system (Madathil et al. 2013, Homsi et al. 2010); although following mTBI there is variability in the time course of responses, depending on severity, numbers and inter-injury interval (Bolton \& Saatman 2014, Luo et al. 2014, Shitaka et al. 2011). Experimental studies investigating repeated mTBI-induced myelin pathology are limited (Shitaka et al. 2011, Gold et al. 2018); myelin pathology has been demonstrated in human studies of concussion (Cubon et al. 2011, Wright et al. 2016). Additionally, while $\mathrm{Ca}^{2+}$ overload and oxidative stress have been implicated as drivers of pathology following moderate-severe TBI, based largely upon measures of cerebral blood flow and metabolic indicators (Barkhoudarian et al. 2016, Vagnozzi et al. 2005), direct measures of oxidative damage in mTBI are lacking. A demonstration of oxidative damage following one or more mTBI would indicate that anti-oxidant therapies may be of use following these injuries.

Using an adapted weight-drop model of closed-head injury (Kane et al. 2012, Marmarou et al. 1994) delivered to adult female PVG rats, we have previously demonstrated differential outcomes between animals experiencing sham, 1, 2 or 3 mTBI on 3 consecutive days, assessed acutely at day 4 (Yates et al. 2017). Specifically, we found cognitive deficits and lipid peroxidation following 2 but not 3 mTBI (Yates et al. 2017). Here we utilise the same model to analyse chronic mechanisms of pathology comparing differences following 1, 2, or $3 \mathrm{mTBI}$, and assess outcomes chronically at 3 months after the first injury. We assess behaviour, together with oxidative damage, microglial and astrocyte responses, as well as myelin ultrastructure (Payne et al. 2012), in various regions of the brain known to be susceptible following repeated mTBI. We hypothesised that there would be a cumulative effect of increasing numbers of mTBIs on chronic behavioural deficits, oxidative damage,

This article is protected by copyright. All rights reserved. 
astrocyte reactivity, neuroinflammation, and myelin structural changes in this in vivo rat model of repeated mTBI.

\section{Materials and Methods}

\section{Animals}

Sixty adult female Piebald Viral Glaxo rats (160 - 200 g, RRID:RGD_737658) aged approximately 3 months were obtained from the Animal Resources Centre (Murdoch, Western Australia). Animals were housed 2 or 3 to a cage with ad libitum access to chow and water, under temperaturecontrolled conditions on a 12 hour (h) light/dark cycle. This study was approved by The University of Western Australia's Animal Ethics Committee (Approval Number RA/3/100/1366) and conducted in accordance with the National Health and Medical Research Council Australian Code of Practice for the care and use of animals for scientific purposes. The study was not pre-registered.

\section{Experimental groups and study design}

A randomised 4-animal block allocation was employed to assign animals to one of four experimental groups ( $n=15$ animals/group): sham, 1, 2 or 3 mTBls. Animals were then numbered 1 to 60 such that for each sequential set of 4 animals, 1 animal in each set was allocated into each experimental group. Animals were then consecutively housed 3 to a cage. Sample size was based upon previous experience (Yates et al. 2017) indicating numbers of animals required for statistical power of behavioural assessments. Injuries or sham procedures were administered $24 \mathrm{~h}$ apart over 3 days (see Figure 1A for experimental design). To control for anaesthesia and analgesia exposure, sham animals received identical procedures excluding injury and injured rats underwent sham procedures on days not injured such that all animals received equal exposure to anaesthetic. Three months after the last $\mathrm{mTBI}$ or sham procedure, each animal underwent 5 days of behavioural testing, hereafter

This article is protected by copyright. All rights reserved. 
referred to as days (D) $1-5$. Following euthanasia, animals numbered 1 to 10 for each group, as per the initial random allocation and numbering process, were analysed using immunohistochemistry ( $\mathrm{n}$ = 10/group); animals numbered 11 to 15 were analysed using transmission electron microscopy (TEM) ( $n=5 /$ group). Note that one animal in the 1 mTBI group died overnight following injury; this was likely due to respiratory depression, however an autopsy was not performed. For replacement, an additional animal was randomly selected from animals received into the facility at the same time.

\section{mTBI protocol}

Rats were subjected to an impact-acceleration model of closed-head repeated mTBI using a custommade weight-drop device (Figure 1B; Northeast Biomedical Inc. MA, USA), as previously described in full (Yates et al. 2017). In brief, a mTBI was delivered by dropping a $250 \mathrm{~g}$ weight fitted with a restraining device to prevent re-hits through a $1 \mathrm{~m}$ high metal guide tube ( $3 \mathrm{~cm}$ inner diameter). The calculated velocity of the impact-acceleration injury was $4.43 \mathrm{~m} / \mathrm{s}$. Similar speed parameters have been used in other studies utilising a Controlled Cortical Impactor (Itzkovich et al.) or closed-head mTBI (Deford et al. 2002, Shitaka et al. 2011, Luo et al. 2014). The mean impact force using this model is $450 \pm 24 \mathrm{~N}$ and the mean head vertical linear velocity is $1.37 \pm 0.21 \mathrm{~m} / \mathrm{s}$ (Yates et al. 2017). Rats were anaesthetised with $4.5 \%$ Isofluorane in $100 \%$ oxygen $(4 \mathrm{~L} / \mathrm{min}$, Attane, Piramal Healthcare, Boise, Idaho; supplied by Provet, Malaga, WA, Australia: Cat\# 60307-120-25) for approximately 1 - 2 min, maintained through a nose cone to minimize animal suffering during the procedure, as described previously (Yates et al. 2017), then placed prone on a delicate task wiper (Kimwipes, Kimberley-Clark Inc., Irving, Texas: Cat\# KCK34120), securely suspended over the central hole in the apparatus stage of the device. The impactor tip $\left(5 \mathrm{~mm}^{2}\right)$ was lowered to the impact site (Figure 1B), aligned along the midline at lambda, determined by locating the interaural line. The impactor tip was then dipped in non-toxic paint before retraction to the top of the guide tube, to allow for impact visualisation. After confirming absence of the foot withdrawal reflex, the weight was released. Upon

This article is protected by copyright. All rights reserved. 
impact, the rat head readily penetrated the Kimwipe, involving a $180^{\circ}$ horizontal rotation of the body onto soft foam padding $15 \mathrm{~cm}$ below the platform. All rats recovered consciousness following injury in less than $5 \mathrm{~min}$. Following each $\mathrm{mTBI}$ or sham procedure, rats were immediately placed on a heating pad $\left(37^{\circ} \mathrm{C}\right)$ and administered analgesia $(4 \mathrm{mg} / \mathrm{kg}$ carprofen subcutaneously; Carprieve, Norbrook Laboratories, supplied by Provet, Malaga, WA, Australia: Cat\# CARP-I-20) to minimize post-procedural suffering. Animals were visually monitored for seizures (none observed) and overt signs of skull fracture were assessed upon euthanasia at 3 months after mTBI (none observed). Visible damage to the cortex was observed for one animal that received $3 \mathrm{mTBI}$, with slight damage to the surface of the left cortex and focal bleeding. Time to spontaneously reorient from a supine to prone position (righting reflex) was measured to assess duration of unconsciousness and impact locations were recorded to ensure consistency.

\section{Behavioural Assessments}

Assessment of neurological function: Gross neurological motor function was evaluated using a neurological severity score (NSS), adapted from a study of moderate closed-head TBI in rats (Shapira et al. 1988). The NSS is derived from a 15 -item checklist assessing: ability to move, seeking behavior, prostration, flexion of hind limbs when raised by the tail, hemiplegia, limb reflexes, startle reflex and inability to walk straight. Additionally, functional tests assessed: failure to balance for $60 \mathrm{~s}$ on platforms $(2 \times 2 \mathrm{~cm}$ and $5 \times 5 \mathrm{~cm})$ and a round beam, and inability to cross a beam $(1,2$ and $3 \mathrm{~cm})$ without exhibiting foot faults. Investigators were blinded to the injury status of each animal and gave each animal a score of either 1 (yes) or 0 (no) on each assessment, summing all scores for a maximum possible NSS of 22 , such that scores increased with severity of dysfunction. No animals were excluded from analysis.

This article is protected by copyright. All rights reserved. 
Assessment of motor function: Slight modifications were made to the skilled ladder rung walking test (Metz \& Whishaw 2009), hereafter referred to as the ladder walk. The ladder walk was used to reveal subtle motor impairments by assessing limb placement and inter-limb coordination during walking. Briefly, the testing apparatus consisted of 2 clear Plexiglass walls (19 cm height, $1 \mathrm{~m}$ length), separated by a series of metal rungs (10 $\mathrm{cm}$ length, $3 \mathrm{~mm}$ diameter at $2 \mathrm{~cm}$ intervals). On D1, following NSS testing, rats completed 5 consecutive trials for acclimation with rungs placed in a regular alternating pattern. On D2, following a single trial with rungs placed in the regular alternating pattern, the position of the rungs was altered to increase task difficulty and prevent both anticipatory and learning effects; there were 5 different rung patterns that each rat walked across. The ladder walk was recorded with a video camera (Canon HG10), positioned in the mid-frontal plane at a slight ventral viewing angle to allow for simultaneous recording of limb placement. Forelimb and hindlimb movements were analysed for foot faults and placement accuracy (Metz \& Whishaw 2009). The numbers of errors, where the foot completely missed the rung, or corrections, where the foot was placed on the rung after a minor slip, were counted by a single investigator blinded to group identity and expressed as a percentage of the total number of steps for all 6 sets; no animals were excluded from analysis.

Assessment of cognitive function: Spatial learning and memory were evaluated on D3 - 5, using standard Morris Water Maze (MWM) procedures (Morris 1984). The animals were not trained in the MWM before $\mathrm{mTBI}$, to ensure testing was of mTBI-induced learning impairments. Each rat underwent 3 testing paradigms over 3 days: acquisition, reversal and probe trial respectively. The platform was hidden in the south west (SW) quadrant during acquisition training on D3 and moved to the opposite north east (NE) quadrant during reversal training on D4. This difference enabled assessment of the rats' ability to extinguish and re-learn the location of the platform (Vorhees \& Williams 2006). On both D3 and D4, rats were allowed to swim freely for approximately $120 \mathrm{~s}$ or until they found the hidden platform. A total of 4 blocks of 4 trials were conducted, with an interThis article is protected by copyright. All rights reserved. 
block interval of approximately $15 \mathrm{~min}$. Animals failing to find the platform were guided to it, and remained there for approximately $15 \mathrm{~s}$. For each trial, rats were placed into the MWM in pseudorandom order, at 1 of 4 insertion points (N, S, E, W). Finally, the platform was removed during the probe trial on D5 and rats were allowed to swim for approximately $90 \mathrm{~s}$ to evaluate memory of the last remembered platform location. Following each trial, the rats were towel-dried and left to warm under a heating lamp (50 W). All trials were video recorded (iSpy, www.ispyconnect.com) using a ceiling-mounted webcam (C270 Logitech) connected to a laptop. Data were collected using ANYMaze (Stoelting Co.) video tracking software, with outcomes including, but not limited to: swim speed; distance travelled; time spent in each quadrant; latency to find the platform; distance from platform; and platform crossings (number of times the animal traverses the precise location where the platform was previously positioned). Analyses were conducted by investigators blinded to group identity; no animals were excluded from analysis.

\section{Immunohistochemistry assessments}

Following behavioural assessments, all rats were sacrificed using Lethabarb $(500 \mathrm{mg} / \mathrm{kg}$ intraperitoneal sodium pentobarbital, Virbac; supplied by Provet, Malaga, WA, Australia: Cat\# Leth1). Animals for immunohistochemical assessments $(n=40)$ were transcardially perfused with $0.9 \%(\mathrm{v} / \mathrm{v})$ saline, then $4 \%(\mathrm{w} / \mathrm{v})$ paraformaldehyde (Acros; supplied by Life Technologies, Thermo Fisher Scientific, Australia: Cat\# ACR169650010), in 0.1M phosphate buffer (pH 7.2). Brains were removed and post-fixed for $24 \mathrm{~h}$ in $4 \%$ paraformaldehyde, then cryoprotected for at least 3 days in $15 \%(\mathrm{w} / \mathrm{v})$ sucrose with $0.1 \%(\mathrm{w} / \mathrm{v})$ sodium azide (Sigma-Aldrich, Castle Hill, NSW, Australia: Cat\# S2002-25g), in phosphate buffered saline (PBS) to allow storage at $4^{\circ} \mathrm{C}$. Brains were hemisected and embedded in optimal cutting temperature compound (OCT; Tissue-Tek, ProSciTech, Townsville, Queensland, Australia: Cat\# IA018) before cryosectioning. The right hemisphere of each animal was sectioned medio-laterally at $25 \mu \mathrm{m}$ and serially collected into multiwell plates containing PBS with

This article is protected by copyright. All rights reserved. 
$0.1 \%(\mathrm{w} / \mathrm{v})$ sodium azide and stored at $4^{\circ} \mathrm{C}$. Free-floating sagittal brain sections were used for immunohistochemical analysis using established procedures (Fitzgerald et al. 2009).

Fluorescence immunohistochemistry: For each animal, 2-4 serial sections that clearly encompassed areas of the hippocampus and allowed easiest delineation of the dentate hilus and corpus callosum were selected, washed twice in PBS and mounted onto Superfrost ${ }^{\circledR}$ Plus slides (Thermo Fisher Scientific, Australia: Cat\# MENSF41296SP). Sections were air dried, rehydrated in PBS (pH 7.2 - 7.4) for $2 \times 5$ min and incubated overnight at $4^{\circ} \mathrm{C}$ in PBS containing $0.2 \%(v / v)$ Triton-X 100 (PBST) (Sigma Aldrich, Castle Hill, NSW, Australia: Cat\# X100-100ML), 5\% (v/v) normal donkey serum (Sigma Aldrich, Castle Hill, NSW, Australia: Cat\# D9663-10mL) and primary antibodies detecting: 8-hydroxy2'-deoxyguanosine (mouse 1:500 80HdG; Abcam, Ab62623, RRID:AB_940049) or astrocyte glial fibrillary acidic protein (mouse 1:500 GFAP GA-5 clone; Merck MAB360 - RRID not available). The following day sections were washed with PBS $(3 \times 5 \mathrm{~min})$, incubated for $2 \mathrm{~h}$ at room temperature with Hoechst nuclear stain $(0.5 \mu \mathrm{g} / \mathrm{ml}$, Invitrogen) and species-appropriate secondary antibodies for antibody binding visualisation: AlexaFluor ${ }^{\circledast} 488$ conjugated goat anti-mouse for $80 \mathrm{HdG}$ and AlexaFluor ${ }^{\circledR} 647$ conjugated donkey anti-mouse for GFAP (1:500 in PBST, Thermo Fisher Scientific, Australia: RRID:AB_10892893 and RRID:AB_162542 respectively). Sections were washed with PBS for $2 \times 5 \mathrm{~min}$, air dried and cover-slipped using Fluoromount-G (Southern Biotech). To ensure uniformity of procedures, all sections for each antibody were immunostained simultaneously.

Immunohistochemistry for light microscopy: $25 \mu \mathrm{m}$ free floating sections (1-2 per animal) were transferred to fresh multiwell plates, washed twice in PBS ( $\mathrm{pH} 7.2$ ) for 5 min each and then PBST. To inhibit endogenous peroxidases, sections were incubated with $0.3 \%(\mathrm{v} / \mathrm{v}) \mathrm{H}_{2} \mathrm{O}_{2}$ in methanol for 45 min. Sections were again washed for $2 \times 5$ min with PBST and incubated overnight at $4^{\circ} \mathrm{C}$ in PBST and primary antibody IBA1 (goat 1:1000; Abcam, Ab5076, RRID:AB_2224402) detecting reactive

This article is protected by copyright. All rights reserved. 
resident microglia/macrophages. The following day sections were washed with PBS $(3 \times 5 \mathrm{~min})$ and incubated in biotinylated anti-goat secondary (1:250 in PBS; BioAffinity; supplied by Vector Laboratories, Burlingame, CA: Cat\# BA-9500) for $2 \mathrm{~h}$ at room temperature, then again washed with PBS $(3 \times 5 \mathrm{~min})$. IBA1 staining was visualised according to manufacturer's instructions using 3,3'diaminobenzadine (DAB) as chromogen (Vectastain ABC Kit, Vector Laboratories: Burlingame, CA: Cat\# PK-4000). The chromogen reaction was previously optimised to determine the 10 min DAB reaction time that would maximise IBA1-positive microglial cell staining while minimising background. Sections were washed with PBS and mounted onto Superfrost ${ }^{\circledR}$ Plus glass slides, air dried, cleared in Xylene and cover-slipped using Depex (Gurr; Supplied by Merck, Bayswater, VIC, Australia: Cat\# 36125). To ensure uniformity of procedures, the sections were all processed at the same time with consistent concentrations of reagents, incubation conditions and development times. Negative controls (no primary antibody) were processed alongside experimental sections.

Image collection: Immunostained brain sections were visually examined prior to experimenter blinding, to determine regions of interest based on differential staining patterns between experimental groups. In addition, regions including the splenium of the corpus callosum and retrosplenial cortex (Figure 2A) were chosen a priori, regardless of staining pattern, based upon the location of injury and previous literature (Wright et al. 2016). Choice of areas of analysis was also dependent upon a staining pattern that enabled analysis. For example, IBA1 immunoreactivity using DAB was too intense in the molecular and cellular layer of the hippocampus to count IBA1 positive microglia. Consistently defined areas within each region of interest were identified and following blinding of the investigator to animal identity, fluorescent images were generated using a confocal Nikon c2 mounted on an upright Ni-E microscope (Nikon Corporation) controlled by NIS elements software (version 4.3). Images were taken in a series of z stacks $(0.5 \mu \mathrm{m}$ optical section thickness; approximately 13 images per stack) at 10x magnification, with the exception of the corpus callosum

This article is protected by copyright. All rights reserved. 
which was captured at $20 \times$ magnification. Single fields of view were captured for all regions, for each of the 2-4 serial sections. Images of DAB treated sections were acquired using an automated slide scanner (Scanscope ${ }^{\circledR} \mathrm{XT}$, Aperio), capturing entire $25 \mu \mathrm{m}$ sagittal sections of the brain at a magnification of $40 x$; digital analysis was performed at $10 \times$ to ensure capture of suitable areas of interest. All images were captured with consistent acquisition parameters. Note that the brain from one animal was fractured during the snap freezing process and was imaged where possible, in small intact regions of interest.

Image analysis: Immunointensity analyses were performed for 8OHdG and GFAP using FIJI (version Mac OS X) analysis software by an investigator blinded to image identity. In order to ensure optimal visualisation, a single image in the z plane (selected based on optimal image clarity) was used for each analysis. To semi-quantitatively assess immunoreactivity, consistent areas of interest were outlined, guided by structural features in The Rat Brain in Stereotaxic Coordinates, 7th Edition (Paxinos \& Watson 2014). Threshold intensities were arbitrarily defined to capture positive cells of interest while minimising background staining and were kept constant for all images. Mean intensity above the set threshold was assessed, together with the area above the set threshold, corrected for the size of the area analysed. Values within each defined region in all sections analysed were averaged to generate a single mean value for each animal $(n)$ for that region, and this was used for statistical analyses.

For density analysis of IBA1 positive microglia, images were viewed by an investigator blinded to animal identity and consistent areas selected in Aperio ImageScope (version 12.0, Inc.) and analysed in FIJI (version Mac OS X). A custom-made macro was used, designed to detect IBA1 immunoreactivity of a size that reflected a cell somata, and above an arbitrarily assigned and consistent threshold intensity that excluded the majority of cell processes. The macro generated

This article is protected by copyright. All rights reserved. 
data for area above threshold as well as the number of cells, allowing calculation of IBA1+ cell size. Analyses were conducted on images of the posterior cortex and splenium of the corpus callosum, averaging outcomes from 1-2 sections per animal. Initial pilot analyses were undertaken on the dentate hilus, brainstem and cerebellum, but due to the differential nature of DAB staining, a consistent threshold intensity could not be determined (data not shown).

In a few instances, there was a reduction in n's for some groups. For immunointensity analyses, some regions of interest were not consistent between groups and were therefore not analysed. Additionally, there were some errors generated in FIJI during immunointensity analyses likely due to variability of staining that were not detectable within the range of arbitrary thresholds set in the macro. Similarly for IBA1 DAB analyses, blurred images from the scanning process or errors generated in FIJI during analyses (likely due to variability of staining as a consequence sections curling in the well during staining), led to a reduction in n's for some groups (see Figure legends for final n's).

\section{Transmission electron microscopy}

Tissue preparation: Following euthanasia, animals for ultrastructural analyses $(n=20)$ were transcardially perfused with $0.9 \%(\mathrm{w} / \mathrm{v})$ saline, then $2 \%(\mathrm{w} / \mathrm{v})$ paraformaldehyde/2.5\% $(\mathrm{w} / \mathrm{v})$ glutaraldehyde/2\% (w/v; Electron Microscopy Sciences, Hatfield, PA: Cat\# 16360) sucrose in 0.1M phosphate buffer ( $\mathrm{pH}$ 7.2). Brains were placed into $0.13 \mathrm{M}$ Sorenson's phosphate buffer ( $\mathrm{pH} 7.2)$ for storage at $4{ }^{\circ} \mathrm{C}$. Small blocks of the splenium of the corpus callosum were cut; post-fixed in $1 \%$ osmium (Electron Microscopy Sciences; supplied by ProSciTech, Townsville, Queensland, Australia: Cat\# C011) for 90 min with shaking. The tissue was processed using a Lynx processor, dehydrating through an ethanol series to propylene oxide, infiltrating with resin into Araldite Procure mixture (ProSciTech Townsville, Queensland, Australia: Cat\# C039). Epoxy resin-embedded brain segments

This article is protected by copyright. All rights reserved. 
were cured for $24 \mathrm{~h}$ at $60^{\circ} \mathrm{C}$ and serially sectioned on an ultramicrotome (LKB Nova, Bromma, Sweden). Semi-thin $(1 \mu \mathrm{m})$ transverse sections were deplasticised with saturated $\mathrm{NaOH}$ in $70 \%(v / v)$ ethanol and stained for $15-30 \mathrm{~s}$ at $95^{\circ} \mathrm{C}$ in aqueous $5 \%$ toluidine blue in $1 \%$ borax. The transverse nature of the sections was confirmed by the circular appearance of the axons. Low-power light micrographs of entire sections were taken at $20 \times$ magnification to determine cytoarchitectural boundaries for TEM analysis. Transverse ultra-thin sections $(100 \mathrm{~nm})$ of the splenium of the corpus callosum were then cut using a diamond knife, mounted onto copper support grids $(3.05 \mathrm{~mm})$ and post-stained with uranyl acetate and lead citrate.

Image collection: Ultra-thin sections were viewed using a TEM (JEOL2100; Tokyo, Japan). Images were generated using the attached Olympus Megaview III digital camera (Orius; Gatan, Pleasanton, CA) and acquired using Olympus iTEM Soft Imaging Solution software. A copper grid was used to define consistent regions of analysis in all sections assessed. Images were taken at a primary magnification of $4000 \times$ in the most central portion of the splenium of the corpus callosum, with 64 high power images (final magnification $15000 \times$ ) generated in a serial fashion to create a photomontage such that myelinated axons of all sizes were captured. Individual images were stitched together to create one photomontage per animal ( $n=5 /$ group), using the MosaicJ Stitching plugin in FIJI analysis software, encompassing approximately 1500 axons per animal.

Ultrastructural analysis of myelinated axons: Given the pronounced rostrocaudal topography of unmyelinated axons in the corpus callosum and lack of clarity of unmyelinated axons, only myelinated axons were quantified. TEM photomontages were first used to count 300 myelinated axons in a consistent location within a montage of images (e.g. Figure 2B). The myelin ensheathing each of these axons was observed to fall into 1 of 5 categories: normally-myelinated, marginallydecompacted, partially-decompacted, paranodal or aberrant profiles (Figure 2C), modified from

This article is protected by copyright. All rights reserved. 
previously descriptions (Payne et al. 2012), as described below and by the addition of classification of paranodal profiles where there was a large axolemmal space and aberrant profiles as in (Reeves et al. 2012). Myelinated axons were classified as 'normally-myelinated' if axons were enwrapped with compact, electron-dense myelin and less than $10 \%$ of the circumference of myelin had separated into distinct layers of lamellae. Axons enwrapped with myelin that was separated for $10-35 \%$ of the circumference were classified as marginallydecompacted, and axons enwrapped with myelin that was separated for $>35 \%$ of the circumference were classified as partially-decompacted. Axon diameter, fibre diameter, myelin thickness and $\mathrm{g}$ ratio (axon diameter/ fibre diameter) (Franklin \& Ffrench-Constant 2008) were calculated to assess subtle changes in myelin ultrastructure following single and repeated mTBI. Minimum axon, fibre and myelin measurements (Figure 2D) were used to avoid skewing of the data due to axons projecting at oblique angles through the tissue section. Where myelin was not decompacted, minimum fiber diameter was measured at the same point as minimum axon diameter, and incorporated the axon diameter, axolemmal space and myelin sheath. Minimum myelin thickness was averaged from two areas of compact myelin (Figure 2D). All analyses were conducted using Fiji (version Mac OS X) software by a single investigator blinded to animal identity and no animals were excluded from analysis.

\section{Statistical analyses}

All data were collected in Excel (version 15.18) and statistical analyses were performed using the statistical program SPSS Statistics (version 21.0), with $p \leq 0.05$ required for statistical significance. Tukey's post-hoc test was used to compare mTBI groups to each other to assess the effects of increasing numbers of mTBls and Dunnett's post-hoc test was used to compare each mTBI group to sham, to assess the individual effects of 1,2 and $3 \mathrm{mTBI}$. Initial analyses of MWM data in the statistical program $\mathrm{R}$, version 3.5.0 using packages ez and plyr, were used to guide post hoc testing in

This article is protected by copyright. All rights reserved. 
SPSS. Normality was assessed using Q-Q plots and homogeneity of variance was assessed using Levene's test.

Rodent behavioural assessments: As the NSS comprises a summation of ordinal measures, KruskalWallis $\mathrm{H}$ test was used as a ranked-sum approach to evaluate the significance of injury on gross neurological function. Individual tests making up the NSS were scored using a binary system $(0,1)$. Categorical data were analysed using Pearson's Chi-squared test with adjusted residuals to assess any significant associations between the outcome and experimental groups. For the MWM probe trial, 2-way ANOVA was conducted to assess main effects of injury on percentage of time spent in the target quadrant and distance from the target platform compared to the opposite quadrant and platform, respectively, and 1-way ANOVA used to assess other outcomes.

Immunohistochemistry and TEM assessments: Data were analysed using 1-way ANOVA, with posthoc tests conducted as appropriate. For ANOVAs, F values, degrees of freedom (dF) of the numbers of groups assessed and p-values are reported. Additionally, a modified Kolmogorov-Smirnov (KS) test was used to determine significant differences between distributions of myelin ultrastructural outcomes. A permutation analysis (re-sampling without replacement, repetitions $=5000$ ) assumes no association between experimental groups and outcome measures. Conducted at the level of animals to avoid pseudo-replication, the permutation assessed for non-random associations, determining the probability of obtaining a test statistic in the randomised samples that is more extreme than the test statistic generated by the measured dataset. Data analysed using the modified KS test were presented as cumulative frequency graphs, made using Excel (version 15.18). All other graphs were made using GraphPad Prism (version 7.0a).

This article is protected by copyright. All rights reserved. 


\section{Results}

The rodent TBI model generates a mild injury

Here we confirm the mild nature of the injury and absence of pronounced ill effects (Dewitt et al. 2013). There was no evidence of seizures or respiratory arrest regardless of the number of mTBls an animal received. The rate of mortality following sham, 1, 2 or $3 \mathrm{mTBI}$ was $0 \%$ and all rats recovered consciousness following injury in less than $5 \mathrm{~min}$, with no differences in the time to righting between experimental groups on days $1(F=0.292, d f=3, p=0.831), 2(F=1.130, d f=3, p=0.345)$ or 3 after injury $(F=0.725, d f=3, p=0.541$ ) (data not shown). Skulls were examined for overt signs of fracture immediately following each $\mathrm{mTBI}$ and the absence of skull fracture was confirmed upon euthanasia at 3 months following $\mathrm{mTBI}$. Brains of $\mathrm{mTBI}$ rats were typically indistinguishable from brains of sham rats on gross pathological examination (Figure $3 \mathrm{~A}$ ) and no extracerebral haemorrhages were detected macroscopically. No cortical damage beneath the impact was observed (Figure 3B), with the exception of 1 animal that received 3 mTBls and exhibited minor damage to the left cortex with focal bleeding. Blood was observed in the lateral ventricles in 5 of the injured animals (11.1\%); none in shams.

In order to ascertain the severity of impact and assess whether increasing numbers of mTBI affected gross neurological function, animals underwent a suite of tests assessing motor, sensorimotor and vestibulomotor domains, comprising a total NSS score. NSS was conducted at both acute and chronic (D1) time points; acutely to assess the immediate severity of the injury and chronically to determine the presence of long lasting deficits in these functional outcomes. There were no significant differences in the distributions of NSS scores acutely (data not shown) or at 3 months between sham, 1,2 or 3 mTBI groups $\left(\chi^{2}=(3, N=60)=4.108, p=0.250\right.$; Figure $\left.4 A\right)$. A ladder walk on D2 was used as an additional measure of motor function; the percentage of forelimb foot placements that missed the rungs of the ladderwalk (errors) or that required correction were not significantly altered

This article is protected by copyright. All rights reserved. 
as a consequence of $\mathrm{mTBI}$ (errors: $\mathrm{F}=0.197, \mathrm{df}=3, \mathrm{p}=0.898$; corrections: $\mathrm{F}=2.605, \mathrm{df}=3, \mathrm{p}=$ 0.061; Figure 4B); hindlimb outcomes were more variable and also not statistically significant (data not shown).

\section{Chronic memory deficits following 1 and 2 but not 3 mTBI}

The MWM was used to evaluate the effects of increasing numbers of mTBI on spatial learning and memory. Data refer to 'opposite' and 'target' in reference to the first and last known location of the platform during acquisition (D3) and reversal (D4) trials respectively (Figure 5A). Mean swim speeds were assessed to ensure fatigue or functional motor impairment were not contributing to the assessment of cognitive function. All animals exhibited similar mean swim speeds (acquisition: $F=$ $0.350, d f=3, p=0.789$; reversal: $F=0.601, d f=3, p=0.617$, Figure $5 C ;$ probe: $F=0.219, d f=3, p=$ 0.883, data not shown). The mean escape latencies (time taken to find the platform) did not change within blocks of trials as a result of $\mathrm{mTBl}$, for either the acquisition or reversal trials (acquisition: $\mathrm{F}=$ 0.190, $d f=3, p=0.902 ;$ reversal: $F=0.761, d f=3, p=0.520$, Figure $5 B$ ).

During the probe trial (D5), there were no significant differences between experimental groups in the percentage of time spent in either the target $(F=1.086, d f=3, p=0.364$; Figure $5 D)$ or opposite quadrants $(F=0.292, d f=3, p=0.831$; Figure $5 D)$. However, 2-way ANOVA revealed a significant main effect of the quadrant $(F=171.2, d f=3,49, p \leq 0.0001$, Figure $5 D)$, with all animals spending a significantly higher percentage of time in the target compared to the opposite quadrant $(p \leq 0.0001)$, indicating that all animals were successful in 're-learning' the new platform position on D4. Time taken to reach the target platform location was not significantly different between experimental groups $(F=2.069, d f=3,56, p=0.115$, Figure $5 E)$, although some rats with $1(n=3)$ or $2(n=1) m T B I$ were unable to locate the target platform within $90 \mathrm{~s}$. In contrast, there were significant differences between experimental groups in target $(F=4.337, d f=3,56, p=0.008$, Figure $5 F)$ but not opposite

This article is protected by copyright. All rights reserved. 
platform crossings $(F=1.308, d f=3,56, p=0.281$, Figure $5 F)$. For target platform crossings, post-hoc analysis indicated a significant effect of $1(p=0.014)$ and 2 mTBI $(p=0.011)$ compared to sham, suggesting that rats with 1 or 2 mTBI were impaired in their ability to remember the re-learned task. In contrast, the significant main effect of quadrant $(F=61.83, d f=1,56, p<0.0001)$ on platform crossings revealed that the animals crossed the target platform more times than all experimental groups crossed the opposite platform ( $p \leq 0.0001)$, indicating the rats were successful in reversing their learning.

Microglial hypertrophy in the cortex but not the dentate hilus following $1 \mathrm{mTBI}$ only

The effect of increasing numbers of mTBI was investigated in the dentate hilus of the hippocampus, using immunohistochemistry to detect microglia, astrocytes and $80 \mathrm{HdG}$, an indicator of oxidative damage to DNA. Microglia are a recognised component of the injury response following mTBI (Homsi et al. 2010) and were also assessed in the cortex, directly below the injury site. The area of DAB immunolabelled IBA1 positive microglial cells was expressed relative to the number of these cells, to provide an estimate of cell size, with the caveat that a small proportion of IBA1 immunoreactivity may be due to cell processes not related to a counted cell. In the cortex, IBA1+ microglia size was significantly different between experimental groups $(F=2.913, d f=3, p=0.049$, Figure 6A, B); post-hoc analyses revealed an increase following 1 mTBI compared to shams $(p=$ 0.030 ), but there were no differences between $1 \mathrm{mTBI}$ and 2 or $3 \mathrm{mTBI}$. IBA1+ microglia cell size in the dentate hilus (Figure 6C) did not change as a consequence of increasing numbers of $\mathrm{mTBI}(\mathrm{F}=$ $0.819, d f=3, p=0.493$, Figure $6 D, E)$. Similarly, no effects of increasing numbers of $m T B I$ on the area of GFAP+ astrocyte immunoreactivity above an arbitrary set threshold were observed in the dentate hilus $(F=0.048, d f=3, p=0.986$, Figure $6 F, G)$. Oxidative damage to DNA was assessed as a first indicator of oxidative responses following increasing numbers of mTBI. 8OHdG immunoreactivity was observed in a cytoplasmic distribution of greater intensity in some cells than others (Figure 6H,

This article is protected by copyright. All rights reserved. 
arrow). The area of greater immunoreactivity was expressed relative to the area above a lower threshold in order to provide a quantitative measure of this feature; however no significant changes were observed $(F=1.329, d f=3, p=0.281$, Figure 61$)$.

\section{Microglial hypertrophy in the corpus callosum following 2 mTBI}

The effects of increasing numbers of mTBI on IBA1+ microglia, GFAP+ astrocytes and 8OHdG immunoreactivity were also assessed in the splenium of the corpus callosum (Figure 7A). Where the dorsal hippocampal commissure was present in the ventral region of the splenium of the corpus callosum, it was excluded from the area of the corpus callosum assessed (Figure 7A inset). IBA1+ microglia size in the splenium of the corpus callosum was significantly different between experimental groups $(F=3.749, d f=3, p=0.021$, Figure 7B); post-hoc analyses revealed increases following 2 mTBI compared to shams $(p=0.013)$. Representative images demonstrate the altered morphology of IBA1+ microglia in the corpus callosum following $2 \mathrm{mTBI}$, with increased numbers of processes (Figure 7C). Initial assessments of IBA1+ cell density using one section per animal also showed a significant increase following 2 mTBI relative to sham (data not shown). GFAP+ astrocyte immunoreactivity was also assessed in the splenium of the corpus callosum and no changes in the area above threshold were observed following increasing numbers of $\mathrm{mTBI}(\mathrm{F}=1.688, \mathrm{df}=3, \mathrm{p}=$ 0.189, Figure 7D). Similarly, no changes in $80 \mathrm{HdG}$ immunoreactivity, indicative of DNA oxidation were seen with increasing numbers of $\mathrm{mTBI}(\mathrm{F}=0.702, \mathrm{df}=3, p=0.560$, Figure $7 \mathrm{E})$.

\section{Axonal and myelin ultrastructure in the corpus callosum following increasing numbers of mTBI}

Axon diameter, fibre diameter, myelin thickness and g ratio were quantified to investigate the effects of increasing numbers of $\mathrm{mTBI}$ on axonal and myelin ultrastructure. Photomontages of TEM images were generated from the central region of the splenium of the corpus callosum. TEM images were stitched together to create a visual montage, enabling myelinated axons of all sizes to be This article is protected by copyright. All rights reserved. 
sampled. Three hundred myelinated axons in a consistent location within the montage were assessed, which according to previous literature (Reeves et al. 2012) accounts for $0.0025 \%$ of the total population of myelinated axons in the corpus callosum. Quantitative analyses were confined to normally myelinated axons, in order to eliminate confounding effects due to myelin decompaction; representative images show the consistency of appearance of normally myelinated axons with increasing numbers of mTBI (Figure 8A).

For normally-myelinated axons, scatter plots show distributions of axon diameter relative to $\mathrm{g}$ ratio and myelin thickness (Figure 8B, C); lines of best fit illustrate the lack of clear differences with increasing numbers of mTBI. 1-way ANOVA for individual outcomes indicated that there were no significant differences in normally-myelinated profiles, between experimental groups in axon diameter $(F=0.325, d f=3, p=0.807)$, g ratio $(F=3.085, d f=3, p=0.057)$ or myelin thickness $(F=$ 2.309, $\mathrm{df}=3, \mathrm{p}=0.115)$. Cumulative frequency distributions were generated and a modified KS test performed to investigate subtle shifts in subpopulations of increasing g ratio, myelin thickness and axon diameter (Figures 8D-F). A shift towards a lower g ratio was observed following 2 mTBI compared to sham, however this was significant at the post-hoc level only ( $p \leq 0.05$, Figure 8D).

\section{Discussion}

In this study we employ an experimental design of repeated mTBI and show that not all indicators of chronic pathology are worse at a chronic time-point, following increasing numbers of mTBI. Microglial size is increased in the cortex following 1 but not 2 or $3 \mathrm{mTBI}$. In addition, microglia in the splenium of the corpus callosum increase in size following only 2 mTBI. Subtle ultrastructural changes to g ratio in the corpus callosum at the post-hoc level reflect the microglia finding following 2 mTBI. Importantly, indicators of cognitive ability mirror the microglial outcomes, with deficits in remembering a learned spatial task following 1 and 2 but not $3 \mathrm{mTBI}$. It is not clear why some indices

This article is protected by copyright. All rights reserved. 
of pathology are not present following higher numbers of $\mathrm{mTBI}$. It is also important to note that this study was conducted in female rats, in order to add important data to the literature of the field, which predominantly reports outcomes in male animals. It is possible that the differential pathology following increasing numbers of $\mathrm{mTBI}$ is sex- and/ or inter-injury interval-dependent, and future studies in both male and female animals are necessary.

In this study, a modified weight-drop impact-acceleration model of closed-head repeated mTBI delivered up to $3 \mathrm{mTBI}$ to the posterior midline of an intact skull. A $250 \mathrm{~g}$ weight delivered at a calculated velocity of $4.43 \mathrm{~m} / \mathrm{s}$ did not result in skull fracture and generally did not result in macroscopic structural brain damage, although blood was observed in the lateral ventricles in $11 \%$ of injured animals only. Other models of single and repeated mTBI have similarly reported an absence of hemorrhages or skull fractures following $3 \mathrm{mTBI}$ at speeds of $4 \mathrm{~m} / \mathrm{s}$ but not $5 \mathrm{~m} / \mathrm{s}$ (Luo et al. 2014), suggesting that suitable parameters were used in this mTBI model to elicit pathology following single and repeated mTBI. Additionally, loss of consciousness for all experimental groups was well within the range representing $\mathrm{mTBI}$ in rodent models (Dewitt et al. 2013); anaesthesia administration was short, to limit confounding effects of anaesthesia on cognitive outcomes, and sham animals also received anaesthesia (Rowe et al. 2013). While deficits in this rodent model were relatively mild and could be potentially be viewed as subconcussive (Turner et al. 2014), the behavioural, pathological and ultrastructural deficits observed are reminiscent of chronic consequences described in single and repeated mTBI as a result of impact in humans (Prasad \& Bondy 2015, Jeter et al. 2013). Indeed, mTBI are characterised by a lack of structural changes on conventional imaging, in the presence of cognitive dysfunction (McCrory et al. 2017). It is possible that the observed variability in data and associated lack of statistically significant differences may be due to the unrestrained injury model employed; there is inherent variability in the impact site (Yates et al. 2017) that is greater than in constrained models. Nevertheless, allowing free movement of the head more closely approximates the majority of human $\mathrm{mTBI}$, including the inherent variability in This article is protected by copyright. All rights reserved. 
initial impact and subsequent outcomes. As such, this study provides insights into detrimental as well as possible compensatory effects following repeated mTBI. Importantly, not all patients who have experienced a small number of mTBls go on to experience mild cognitive impairment and the observed outcomes in this study may reflect clinical observations of variable outcomes following small numbers of mTBI (McCrory et al. 2017).

Animals with 1 or 2 mTBI exhibited chronic memory impairments in the probe trial of the MWM, indicated by a significant reduction in the amount of times the animals crossed the area where the platform was most recently located, compared to sham and 3 mTBI rats. Memory deficits following repeated $\mathrm{mTBI}$ are supported by growing literature in both experimental and human studies. Persisting memory impairments in animal models have been reported following 2 (Cheng 2013, Shitaka et al. 2011) and 4 mTBI (Deford et al. 2002) at $24 \mathrm{~h}$ intervals, indicating chronic deficits with short inter-injury intervals (Longhi et al. 2005, Weil et al. 2014, Mannix et al. 2013, Meehan et al. 2012) and increased number of mTBls (Shultz et al. 2012, Meehan et al. 2012, Mannix et al. 2013). Interestingly, the majority of repeated mTBI studies fail to report chronic cognitive deficits following a single mTBI. However, persisting learning and memory impairments are frequently reported following single (Levin \& Robertson 2013) and repeated mTBI (Guskiewicz et al. 2005) in humans. Additional clinical reports suggest cognitive deficits do not differ among athletes sustaining 1 or 2 mTBIs (Macciocchi et al. 2001). This is the first repeated mTBI study in rats to demonstrate an apparent amelioration of cognitive dysfunction with increasing numbers of mTBls, in this case, following $3 \mathrm{mTBI}$. In contrast, 3 or more $\mathrm{mTBI}$ in humans has been associated with irreversible deficits (Macciocchi et al. 1998). Such a dissonance may be due to the inherent differences in rats and humans with regard to brain structure, and the number of mTBIs and inter-injury interval as well as possible compensatory effects. The key finding in this study is that long-lasting cognitive impairments may not simply be due to a cumulative effect of increasing numbers of mTBIs. While we may speculate that compensatory mechanisms may become operative following $3 \mathrm{mTBI}$ (Zhang et al. This article is protected by copyright. All rights reserved. 
2004, Cho \& So 1989), it is important to note that there was no statistically significant difference in cognitive function between animals following 2 and 3 mTBI and this in itself warrants further investigation. Additionally, longitudinal studies comprising careful and complete monitoring of patients who have experienced one or more $\mathrm{MTBI}$ will be required to ascertain whether cognitive deficits are lessened with an additional injury in humans.

In addition to cognitive deficits, we report increased IBA1+ microglia size following 1 and 2 but not 3 mTBI. It has been reported that following 2 mTBls delivered at $24 \mathrm{~h}$ intervals, prominent acute microglial responses persist in white matter until 7 weeks (Shitaka et al. 2011). Interestingly, when 4 mTBIs were given, exacerbated microglial hypertrophy and increased immunoreactivity were observed at acute (Bolton \& Saatman 2014) and sub-acute (Hylin et al. 2013) but not chronic (Mannix et al. 2013) time-points. Increased microglial cell size has been shown to directly correlate to a higher degree of branching and ramification in the normal rat brain (Kongsui et al. 2014). Therefore, our observed increases in microglial soma size indicate greater ramification and therefore possibly, reduced activation (Miron \& Franklin 2014) in response to a small number of mTBI. It is possible that in addition to IBA1 immunoreactivity, markers more closely tied to phenotypic changes, such as arginase 1 and iNOS (Miron \& Franklin 2014), may provide further insight and reveal subtleties of microgliosis or astrogliosis. However, it is increasingly understood that these markers can be co-expressed and increased in the same cells following injury (Smith et al. 2018), and are therefore not definitive indicators of pro- or anti-inflammatory events.

Activated microglia form extended cytoplasmic processes in direct contact with injured axons to form a potential barrier between healthy and injured tissue, suggesting that microglial activation is a response to axonal damage (Davalos et al. 2005, Shitaka et al. 2011). Myelin debris which is present following mTBI can also stimulate the inflammatory process (Mierzwa et al. 2015, Sullivan et al.

This article is protected by copyright. All rights reserved. 
2013). There is a subtle decrease in g ratio observed following $2 \mathrm{mTBI}$ at the post-hoc level only. It is not clear whether this is contributing to cognitive dysfunction following $2 \mathrm{mTBI}$. It is perhaps more feasible that a chronic inflammatory response underlies the cognitive deficits following 1 and 2 but not $3 \mathrm{mTBI}$ in the current study. Correlations between behavioural performance and axonal and microglial pathology have previously been described and it would be useful to assess axonal pathology as a potential driver of cognitive dysfunction (Shitaka et al. 2011). Taken together with our previous examination of acute effects of 1, 2 or 3 mTBI (Yates et al. 2017), outcomes from a diverse array of measures in two separate cohorts of female animals have demonstrated that there is not necessarily a positive correlation between increasing numbers of $\mathrm{mTBI}$ and increasing deficits and damage. The phenomena is reminiscent of a conditioning effect, where an injury can provide protective effects via a predisposition to faster neural regeneration (Zhang et al. 2004, Cho \& So 1989). Repetitive mTBI can condition the brain such that an additional more severe injury does not lead to expected motor deficits (Allen et al. 2000). Similar effects are also observed following ischemic pre-conditioning (Kato et al. 1991). The mechanisms by which inflammation, ultrastructural changes and cognitive deficits do not remain elevated following a third additional mTBI are not yet known and may differ with brain regions. Future studies comparing potential anti-inflammatory and anti-oxidant responses as well as axonal pathology and other mechanisms following greater numbers of $\mathrm{mTBI}$ at different inter-injury intervals will be required to determine how such differential responses occur.

The current study demonstrates that microglial activation and associated cognitive deficits do not necessarily persist with increasing numbers of mTBI. In light of the high incidence of $\mathrm{mTBI}$ and subsequent risk of repeated $\mathrm{mTBI}$, these novel observations have important implications for future research aimed at understanding the pathology and sequelae of repeated $\mathrm{mTBI}$ and developing effective treatments. Repeated mTBI literature to date has largely focused on mechanisms of damage. However, temporally profiling both damage and endogenous protective mechanisms This article is protected by copyright. All rights reserved. 
together are required to investigate how mechanisms interact to influence either progressive or persisting chronic pathology as well as some apparent resolution. Elucidating the interaction between protective and neurotoxic responses in a range of behavioural, cellular, molecular and ultrastructural outcomes, relative to greater numbers and increased inter-injury intervals of mTBI will be required to inform 'return to play' guidelines and develop therapeutic strategies aimed at ameliorating long-term dysfunction following single and repeated mTBI.

\section{Competing interests}

No competing financial interests exist. M. Fitzgerald is an editor for Journal of Neurochemistry.

\section{Authors' contributions}

MF and NY designed the research. $B F, N Y, S L, M A, M M, H M$ and $C B$ performed the research. BF, NY, $\mathrm{SL}$, and JH analysed the data. BF wrote the manuscript. All authors edited, read and/or approved the final manuscript.

--Human subjects --

Involves human subjects:

If yes: Informed consent \& ethics approval achieved:

$\Rightarrow$ if yes, please ensure that the info "Informed consent was achieved for all subjects, and the experiments were approved by the local ethics committee." is included in the Methods.

ARRIVE guidelines have been followed:

Yes

$\Rightarrow$ if it is a Review or Editorial, skip complete sentence $\Rightarrow$ if No, include a statement: "ARRIVE guidelines were not followed for the following reason:

(edit phrasing to form a complete sentence as necessary).

This article is protected by copyright. All rights reserved. 
$\Rightarrow$ if Yes, insert "All experiments were conducted in compliance with the ARRIVE guidelines." unless it is a Review or Editorial

\section{Acknowledgements}

MF is supported by an NHMRC Career Development Fellowship (APP1087114). We thank Dr Andrew Mehnert for providing guidance with creating photomontages for TEM image analysis, Assoc./Prof Caitlin Wyrwoll for her provision of behavioural analysis software, Emeritus Professor Alan Harvey and Dr Ryan O'Hare Doig for helpful discussions, and Terence McGonigle for technical assistance with image deconvolution.

\section{Open Science Badges}

This article has received a badge for *Open Materials* because it provided all relevant information to reproduce the study in the manuscript. The complete Open Science Disclosure form for this article can be found at the end of the article. More information about the Open Practices badges can be found at https://cos. io/our-services/open-science-badges/.

This article is protected by copyright. All rights reserved. 


\section{Figure Legends}

Figure 1. Schematic of experimental design, injury device and location. (A) All animals received either injury (denoted by star) or sham (denoted by dash) procedures $24 \mathrm{~h}$ apart on days 1,2 and 3 . Behavioural assessments of chronic outcomes were conducted for each animal ( $n=15 /$ group) 3 months after the last injury or sham procedure. Neurological severity score was conducted on day 1 at the chronic time point, ladder walk was conducted on day 2 and Morris water maze paradigms were conducted on days $3-5$. All rats were euthanised for immunohistochemistry $(n=10$ animals/group) or electron microscopy ( $n=5$ animals/group) analyses on day 5 at the chronic time point. (B) mTBI was administered along the midline of the skull approximately at lambda, using a custom-made weight-drop device that allowed unconstrained movement of the head.

Figure 2. Schematic representation of the injury site (denoted by star) relative to regions of interest sampled (labelled) (A.) High-power (4000x) transmission electron microscopy (TEM) images within the central splenium of the corpus callosum were stitched together to create montages (B) which were used for quantification. Decompacted myelin was observed as splitting of myelin lamellae into distinct layers. Length of decompacted myelin was expressed as a percentage of total myelinated axon circumference and used to define myelinated axons into categories of normally-myelinated, marginally-decompacted, partially-decompacted, paranodal or aberrant profiles (C). Features of normally myelinated axons were quantified: minimum axon diameter (shown in pink) excluded the axolemmal space; minimum fibre diameter (shown in blue) was taken at the same point as minimum axon diameter but extended across the axolemmal space and myelin sheath; minimum myelin thickness (shown in green) was averaged from two areas of compact myelin. Scale bar $=1 \mu \mathrm{m}$.

This article is protected by copyright. All rights reserved. 
Figure 3. Gross structural pathology following repeated mTBI. A. No overt damage to brains was observed three months following sham, 1, 2 or 3 mTBI (A) and no obvious signs of cell death were visible following cresyl violet staining (B). Scale bar $=3 \mathrm{~mm}$.

Figure 4. Assessments of gross neurological function and subtle motor function following single and repeated $\mathrm{mTBI}$ at 3 months. Graphs display the median, interquartile range and range for the NSS (total score $=22)(A)$ and the percentage of forelimb placements in error or which involved a correction of limb placement for experimental groups in the ladder walk (B). NSS = neurological severity score. $\mathrm{n}=14-15$ animals/group; 1 sham animal did not engage in the $1 \mathrm{~cm}$ beam task and was therefore removed from analysis for the NSS.

Figure 5. Outcomes of memory function in the Morris water maze following single and repeated mTBI. Location of platform relative to quadrant in the acquisition, reversal and probe trials are shown (A). Time taken to find the platform (escape latency) (B) and swim speed (C) are shown as averages for the four blocks of trials for the acquisition and reversal phases. For the probe trial, the time spent in target and opposite quadrants are compared, showing median, interquartile range and range (D); dotted line indicates chance. Time taken to reach the target platform locations (E) and the number of times animals traversed the precise target platform location $(F)$ are similarly shown; $n=$ 15 animals/group. Statistically significant differences are indicated by $* p \leq 0.05, * * * * p \leq 0.0001$, with bracketed lines indicating significances between groups by ANOVA and straight lines indicating post-hoc outcomes.

Figure 6. IBA1, GFAP and $80 \mathrm{HdG}$ immunoreactivity in cortex and dentate hilus of the hippocampus following single and repeated mTBI. Median, interquartile range and range cell size of IBA1+ microglia in cortex (A) and dentate hilus of the hippocampus (C, D) were expressed in $\mu \mathrm{m}^{2}$; This article is protected by copyright. All rights reserved. 
representative images are in (B) and (E) respectively. Graphs show the median, interquartile range and range area of GFAP immunoreactivity above an arbitrarily set and constant threshold (F); representative images are in (G). $80 \mathrm{HdG}$ immunoreactivity (green) was present in a characteristic cytoplasmic distribution of greater intensity in some cells (arrow) than others (dotted arrow, H). The area above a high threshold designed to capture only the areas of elevated intensity was expressed relative to the area above a lower threshold that captured all immunoreactivity (I). Statistically significant differences are indicated by $* p \leq 0.05 . n=10$ animals/group except for: $n=9$ for IBA1 sham cortex and dentate hilus; $\mathrm{n}=9$ for IBA1 1 mTBI cortex; $\mathrm{n}=8$ for IBA1 2 mTBI cortex and dentate hilus; $\mathrm{n}=9$ for $80 \mathrm{HdG}$ sham. Scale bar $=25 \mu \mathrm{m}$.

Figure 7. IBA1, GFAP and $80 \mathrm{HdG}$ immunoreactivity in the splenium of the corpus callosum following single and repeated $\mathrm{mTBl}$; area analysed is indicated in red in the inset, avoiding the dorsal hippocampal commissure (A). Median, interquartile range and range cell size of IBA1+ microglia in the splenium of the corpus callosum were expressed in $\mu \mathrm{m}^{2}(\mathrm{~B})$, representative images are in (C). Graphs show the median, interquartile range and range area of GFAP immunoreactivity (D) and intensity of $80 \mathrm{HdG}$ immunoreactivity (E) above arbitrarily set and constant thresholds. Statistically significant differences are indicated by * $p \leq 0.05 . n=10$ animals/group except: $n=9$ for IBA1 sham; $\mathrm{n}=7$ for IBA1 $2 \mathrm{mTBI} ; \mathrm{n}=9$ for GFAP $2 \mathrm{mTBI} ; \mathrm{n}=9$ for $80 \mathrm{HdG}$ sham. Scale bar $=100 \mu \mathrm{m} . \mathrm{dhc}=$ dorsal hippocampal commissure; and scc = splenium of corpus callosum.

Figure 8. Ultrastructural analyses of axons and myelin in the splenium of the corpus callosum following single and repeated mTBI. Representative transmission electron microscopy images of axons ensheathed in myelin with differing degrees of decompaction (A). Scatter plots of $g$ ratio (B) and myelin thickness $(C)$ relative to axon diameter are shown, with regression lines superimposed. Cumulative frequency histograms illustrate the distributions of $g$ ratio (D), myelin thickness (E) and

This article is protected by copyright. All rights reserved. 
axon diameter (F) in normally-myelinated axons in experimental groups. Statistically significant differences at the post-hoc level only is indicated by \# $p \leq 0.05 . n=5$ animals/group. Scale bars $=1$ $\mu \mathrm{m}$.

This article is protected by copyright. All rights reserved. 


\section{References}

Allen, G. V., Gerami, D. and Esser, M. J. (2000) Conditioning effects of repetitive mild neurotrauma on motor function in an animal model of focal brain injury. Neuroscience, 99, 93-105.

Barkhoudarian, G., Hovda, D. A. and Giza, C. C. (2016) The Molecular Pathophysiology of Concussive Brain Injury - an Update. Phys Med Rehabil Clin N Am, 27, 373-393.

Blennow, K., Brody, D. L., Kochanek, P. M., Levin, H., McKee, A., Ribbers, G. M., Yaffe, K. and Zetterberg, H. (2016) Traumatic brain injuries. Nat Rev Dis Primers, 2, 16084.

Bolton, A. N. and Saatman, K. E. (2014) Regional neurodegeneration and gliosis are amplified by mild traumatic brain injury repeated at 24-hour intervals. J Neuropathol Exp Neurol, 73, 933-947.

Bolton Hall, A. N., Joseph, B., Brelsfoard, J. M. and Saatman, K. E. (2016) Repeated Closed Head Injury in Mice Results in Sustained Motor and Memory Deficits and Chronic Cellular Changes. PLoS One, 11, e0159442.

Briggs, D. I., Angoa-Perez, M. and Kuhn, D. M. (2016) Prolonged Repetitive Head Trauma Induces a Singular Chronic Traumatic Encephalopathy-Like Pathology in White Matter Despite Transient Behavioral Abnormalities. Am J Pathol, 186, 2869-2886.

Carroll, L. J., Cassidy, J. D., Cancelliere, C. et al. (2014) Systematic review of the prognosis after mild traumatic brain injury in adults: cognitive, psychiatric, and mortality outcomes: results of the International Collaboration on Mild Traumatic Brain Injury Prognosis. Arch Phys Med Rehabil, 95, S152-173.

Cassidy, J., Carroll, L., Peloso, P., Borg, J., von Holst, H., Holm, L., Kraus, J. and Coronado, V. (2004) Incidence, risk factors and prevention of mild traumatic brain injury: results of the WHO Collaborating Centre Task Force on Mild Traumatic Brain Injury. Journal of Rehabilitation Medicine, 28-60.

Cheng, M.-F. (2013) Hypothalamic neurogenesis in the adult brain. Frontiers in Neuroendocrinology, 34, 167-178.

Cho, E. Y. and So, K. F. (1989) Regrowth of retinal ganglion cell axons into a peripheral nerve graft in the adult hamster is enhanced by a concurrent optic nerve crush. Exp Brain Res, 78, 567-574.

Cubon, V. A., Putukian, M., Boyer, C. and Dettwiler, A. (2011) A diffusion tensor imaging study on the white matter skeleton in individuals with sports-related concussion. J Neurotrauma, 28, 189 201.

Dashnaw, M. L., Petraglia, A. L. and Bailes, J. E. (2012) An overview of the basic science of concussion and subconcussion: Where we are and where we are going. Neurosurgical Focus, 33, E5.

Davalos, D., Grutzendler, J., Yang, G., Kim, J. V., Zuo, Y., Jung, S., Littman, D. R., Dustin, M. L. and Gan, W.-B. (2005) ATP mediates rapid microglial response to local brain injury in vivo. Nature Neuroscience, 8, 752-758.

This article is protected by copyright. All rights reserved. 
Deford, S. M., Wilson, M. S., Rice, A. C., Clausen, T., Rice, L. K., Barabnova, A., Bullock, R. and Hamm, R. J. (2002) Repeated mild brain injuries result in cognitive impairment in B6C3F1 mice. Journal of Neurotrauma, 19, 427-438.

Dewitt, D. S., Perez-Polo, R., Hulsebosch, C. E., Dash, P. K. and Robertson, C. S. (2013) Challenges in the development of rodent models of mild traumatic brain injury. Journal of Neurotrauma, 30, 688-701.

Donovan, V., Kim, C., Anugerah, A. K., Coats, J. S., Oyoyo, U., Pardo, A. C. and Obenaus, A. (2014) Repeated mild traumatic brain injury results in long-term white-matter disruption. J Cereb Blood Flow Metab, 34, 715-723.

Fehily, B. and Fitzgerald, M. (2016) Repeated mild traumatic brain injury: potential mechanisms of damage. Cell Transplant.

Fitzgerald, M., Bartlett, C. A., Evill, L., Rodger, J., Harvey, A. R. and Dunlop, S. A. (2009) Secondary degeneration of the optic nerve following partial transection: the benefits of lomerizine. Exp Neurol, 216, 219-230.

Franklin, R. J. and Ffrench-Constant, C. (2008) Remyelination in the CNS: from biology to therapy. Nature Reviews Neuroscience, 9, 839-855.

Giacci, M. K., Bartlett, C. A., Smith, N. M., Iyer, K. S., Toomey, L. M., Jiang, H., Guagliardo, P., Kilburn, M. R. and Fitzgerald, M. (2018) Oligodendroglia Are Particularly Vulnerable to Oxidative Damage after Neurotrauma In Vivo. J Neurosci, 38, 6491-6504.

Gold, E. M., Vasilevko, V., Hasselmann, J., Tiefenthaler, C., Hoa, D., Ranawaka, K., Cribbs, D. H. and Cummings, B. J. (2018) Repeated Mild Closed Head Injuries Induce Long-Term White Matter Pathology and Neuronal Loss That Are Correlated With Behavioral Deficits. ASN Neuro, 10, 1759091418781921.

Government, N. S. W. (2011) Children and Infants - Acute Management of Head Injury. (D. o. Health ed.).

Guskiewicz, K. M., Marshall, S. W., Bailes, J., McCrea, M., Cantu, R. C., Randolph, C. and Jordan, B. D. (2005) Association between recurrent concussion and late-life cognitive impairment in retired professional football players. Neurosurgery, 57, 719-726.

Holbourn, A. H. S. (1943) Mechanics of head injury. The Lancet, 242, 438-441.

Homsi, S., Piaggio, T., Croci, N., Noble, F., Plotkine, M., Marchand-Leroux, C. and Jafarian-Tehrani, M. (2010) Blockade of acute microglial activation by minocycline promotes neuroprotection and reduces locomotor hyperactivity after closed head injury in mice: a twelve-week follow-up study. Journal of Neurotrauma, 27, 911-921.

Huang, L., Coats, J. S., Mohd-Yusof, A. et al. (2013) Tissue vulnerability is increased following repetitive mild traumatic brain injury in the rat. Brain Res, 1499, 109-120.

This article is protected by copyright. All rights reserved. 
Hylin, M. J., Orsi, S. A., Rozas, N. S., Hill, J. L., Zhao, J., Redell, J. B., Moore, A. N. and Dash, P. K. (2013) Repeated mild closed head injury impairs short- term visuospatial memory and complex learning. Journal of Neurotrauma, 30, 716-726.

Itzkovich, M., Gelernter, I., Biering-Sorensen, F. et al. (2007) The Spinal Cord Independence Measure (SCIM) version III: reliability and validity in a multi-center international study. Disability and rehabilitation, 29, 1926-1933.

Jeter, C. B., Hergenroeder, G. W., Hylin, M. J., Redell, J. B., Moore, A. N. and Dash, P. K. (2013) Biomarkers for the diagnosis and prognosis of mild traumatic brain injury/concussion. $J$ Neurotrauma, 30, 657-670.

Kane, M. J., Angoa-Perez, M., Briggs, D. I., Viano, D. C., Kreipke, C. W. and Kuhn, D. M. (2012) A mouse model of human repetitive mild traumatic brain injury. J Neurosci Methods, 203, 4149.

Kato, H., Liu, Y., Araki, T. and Kogure, K. (1991) Temporal profile of the effects of pretreatment with brief cerebral ischemia on the neuronal damage following secondary ischemic insult in the gerbil: cumulative damage and protective effects. Brain Res, 553, 238-242.

Kongsui, R., Beynon, S. B., Johnson, S. J. and Walker, F. R. (2014) Quantitative assessment of microglial morphology and density reveals remarkable consistency in the distribution and morphology of cells within the healthy prefrontal cortex of the rat. J Neuroinflammation, 11, 182.

Levin, H. S. and Robertson, C. S. (2013) Mild traumatic brain injury in translation. Journal of Neurotrauma, 30, 610-617.

Longhi, L., Saatman, K. E., Fujimoto, S. et al. (2005) Temporal window of vulnerability to repetitive experimental concussive brain injury. Neurosurgery, 56, 364-374.

Luo, J., Nguyen, A., Villeda, S. et al. (2014) Long-term cognitive impairments and pathological alterations in a mouse model of repetitive mild traumatic brain injury. Front Neurol, 5, 12.

Macciocchi, S. N., Barth, J. T., Littlefield, L. and Cantu, R. C. (2001) Multiple concussions and neuropsychological functioning in collegiate football players. Journal of Athletic Training, 36, 303-306.

Macciocchi, S. N., Barth, J. T. and Littlefield, L. M. (1998) Outcome after mild head injury. Clinicals in Sports Medicine, 17, 27-36.

Madathil, S. K., Carlson, S. W., Brelsfoard, J. M., Ye, P., D'Ercole, A. J. and Saatman, K. E. (2013) Astrocyte-specific overexpression of insulin-like growth factor-1 protects hippocampal neurons and reduces behavioral deficits following traumatic brain injury in mice. PLoS One, 8, e67204.

Maher, M. E., Hutchison, M., Cusimano, M., Comper, P. and Schweizer, T. A. (2014) Concussions and heading in soccer: a review of the evidence of incidence, mechanisms, biomarkers and neurocognitive outcomes. Brain Inj, 28, 271-285.

This article is protected by copyright. All rights reserved. 
Mannix, R., Meehan, W. P., Mandeville, J. et al. (2013) Clinical correlates in an experimental model of repetitive mild brain injury. Ann Neurol, 74, 65-75.

Marmarou, A., Foda, M. A., van den Brink, W., Campbell, J., Kita, H. and Demetriadou, K. (1994) A new model of diffuse brain injury in rats. Part I: Pathophysiology and biomechanics. $J$ Neurosurg, 80, 291-300.

Martini, D. N. and Broglio, S. P. (2017) Long-term effects of sport concussion on cognitive and motor performance: A review. Int J Psychophysiol.

McAteer, K. M., Corrigan, F., Thornton, E., Turner, R. J. and Vink, R. (2016) Short and Long Term Behavioral and Pathological Changes in a Novel Rodent Model of Repetitive Mild Traumatic Brain Injury. PLoS One, 11, e0160220.

McCrea, M., Broshek, D. K. and Barth, J. T. (2015) Sports concussion assessment and management: future research directions. Brain Inj, 29, 276-282.

McCrory, P., Meeuwisse, W., Dvorak, J. et al. (2017) Consensus statement on concussion in sport-the 5 (th) international conference on concussion in sport held in Berlin, October 2016. Br J Sports Med, 51, 838-847.

Meaney, D. F. and Smith, D. H. (2011) Biomechanics of Concussion. Clinics in Sports Medicine, 30, 1931.

Meehan, W. P., 3rd, Zhang, J., Mannix, R. and Whalen, M. J. (2012) Increasing recovery time between injuries improves cognitive outcome after repetitive mild concussive brain injuries in mice. Neurosurgery, 71, 885-891.

Metz, G. A. and Whishaw, I. Q. (2009) The ladder rung walking task: a scoring system and its practical application. J Vis Exp.

Mierzwa, J. A., Marion, M. C., Sullivan, M. G., McDaniel, P. D. and Armstrong, C. R. (2015) Components of myelin damage and repair in the progression of white matter pathology after mild traumatic brain injury. Journal of Neuropathology and Experimental Neurology, 74, 218-232.

Miron, V. E. and Franklin, R. J. (2014) Macrophages and CNS remyelination. J Neurochem, 130, 165171.

Morris, R. (1984) Developments of a water- maze procedure for studying spatial learning in the rat. Journal of Neuroscience Methods, 11, 47-60.

Mountney, A., Boutte, A. M., Cartagena, C. M. et al. (2017) Functional and Molecular Correlates after Single and Repeated Rat Closed-Head Concussion: Indices of Vulnerability after Brain Injury. J Neurotrauma, 34, 2768-2789.

Namjoshi, D. R., Cheng, W. H., Mclnnes, K. A. et al. (2014) Merging pathology with biomechanics using CHIMERA (Closed-Head Impact Model of Engineered Rotational Acceleration): a novel, surgery-free model of traumatic brain injury. Mol Neurodegener, 9, 55.

This article is protected by copyright. All rights reserved. 
Nichols, J. N., Deshane, A. S., Niedzielko, T. L., Smith, C. D. and Floyd, C. L. (2016) Greater neurobehavioral deficits occur in adult mice after repeated, as compared to single, mild traumatic brain injury (mTBI). Behav Brain Res, 298, 111-124.

Paxinos, G. and Watson, C. (2014) The rat brain in stereotaxic coordinates. 7th Edition, Elsevier Academic Press, San Diego.

Payne, S. C., Bartlett, C. A., Harvey, A. R., Dunlop, S. A. and Fitzgerald, M. (2012) Myelin sheath decompaction, axon swelling, and functional loss during chronic secondary degeneration in rat optic nerve. Invest Ophthalmol Vis Sci, 53, 6093-6101.

Prasad, K. N. and Bondy, S. C. (2015) Common biochemical defects linkage between post-traumatic stress disorders, mild traumatic brain injury (TBI) and penetrating TBI. Brain Res, 1599, 103114.

Prins, M. L., Alexander, D., Giza, C. C. and Hovda, D. A. (2013) Repeated mild traumatic brain injury: mechanisms of cerebral vulnerability. J Neurotrauma, 30, 30-38.

Reeves, T. M., Smith, T. L., Williamson, J. C. and Phillips, L. L. (2012) Unmyelinated axons show selective rostrocaudal pathology in the corpus callosum after traumatic brain injury. $J$ Neuropathol Exp Neurol, 71, 198-210.

Rivara, F. P. and Graham, R. (2014) Sports-related concussions in youth: report from the Institute of Medicine and National Research Council. JAMA, 311, 239-240.

Rowe, R. K., Harrison, J. L., Thomas, T. C., Pauly, J. R., Adelson, P. D. and Lifshitz, J. (2013) Using anesthetics and analgesics in experimental traumatic brain injury. Lab Animal, 42, 286-291.

Selwyn, R. G., Cooney, S. J., Khayrullina, G., Hockenbury, N., Wilson, C. M., Jaiswal, S., Bermudez, S., Armstrong, R. C. and Byrnes, K. R. (2016) Outcome after Repetitive Mild Traumatic Brain Injury Is Temporally Related to Glucose Uptake Profile at Time of Second Injury. J Neurotrauma, 33, 1479-1491.

Shahrokhi, N., Haddad, M. K., Joukar, S., Shabani, M., Keshavarzi, Z. and Shahozehi, B. (2012) Neuroprotective antioxidant effect of sex steroid hormones in traumatic brain injury. Pak $J$ Pharm Sci, 25, 219-225.

Shapira, Y., Shohami, E., Sidi, A., Soffer, D., Freeman, S. and Cotev, S. (1988) Experimental closed head injury in rats: Mechanical, pathophysiologic, and neurologic properties. Critical Care Medicine, 16, 258-265.

Shitaka, Y., Tran, H. T., Bennett, R. E., Sanchez, L., Levy, M. A., Dikranian, K. and Brody, D. L. (2011) Repetitive closed-skull traumatic brain injury in mice causes persistent multifocal axonal injury and microglial reactivity. J Neuropathol Exp Neurol, 70, 551-567.

Shultz, S. R., MacFabe, D. F., Foley, K. A., Taylor, R. and Cain, D. P. (2012) Sub-concussive brain injury in the Long-Evans rat induces acute neuroinflammation in the absence of behavioral impairments. Behav Brain Res, 229, 145-152.

This article is protected by copyright. All rights reserved. 
Smith, N., Giacci, M., Gough, A. et al. (2018) Inflammation and blood-brain barrier breach remote from the primary injury following neurotrauma. Journal of Neuroinflammation, 15.

Sullivan, M. G., Mierzwa, J. A., Kijpaisalratana, C. N., Tang, C. H., Wang, C. Y., Song, C. S.-K., Selwyn, C. R. and Armstrong, C. R. (2013) Oligodendrocyte lineage and subventricular zone response to traumatic axonal injury in the corpus callosum. Journal of Neuropathology and Experimental Neurology, 72, 1106-1125.

Thomsen, G. M., Ma, A. M., Ko, A. et al. (2016) A model of recurrent concussion that leads to longterm motor deficits, CTE-like tauopathy and exacerbation of an ALS phenotype. J Trauma Acute Care Surg, 81, 1070-1079.

Turner, R. C., VanGilder, R. L., Naser, Z. J., Lucke-Wold, B. P., Bailes, J. E., Matsumoto, R. R., Huber, J. D. and Rosen, C. L. (2014) Elucidating the severity of preclinical traumatic brain injury models: a role for functional assessment? Neurosurgery, 74, 382-394; discussion 394.

Vagnozzi, R., Signoretti, S., Tavazzi, B., Cimatti, M., Amorini, A. M., Donzelli, S., Delfini, R. and Lazzarino, G. (2005) Hypothesis of the postconcussive vulnerable brain: experimental evidence of its metabolic occurrence. Neurosurgery, 57, 164-171; discussion 164-171.

Vorhees, C. V. and Williams, M. T. (2006) Morris water maze: procedures for assessing spatial and related forms of learning and memory. Nature Protocols, 1, 848-858.

Weil, Z. M., Gaier, K. R. and Karelina, K. (2014) Injury timing alters metabolic, inflammatory and functional outcomes following repeated mild traumatic brain injury. Neurobiology of Disease, 70, 108-116.

Willer, B. and Leddy, J. J. (2006) Management of concussion and post-concussion syndrome. Curr Treat Options Neurol, 8, 415-426.

Wright, A. D., Jarrett, M., Vavasour, I., Shahinfard, E., Kolind, S., van Donkelaar, P., Taunton, J., Li, D. and Rauscher, A. (2016) Myelin Water Fraction Is Transiently Reduced after a Single Mild Traumatic Brain Injury--A Prospective Cohort Study in Collegiate Hockey Players. PLoS One, 11, e0150215.

Xu, L., Nguyen, J. V., Lehar, M. et al. (2016) Repetitive mild traumatic brain injury with impact acceleration in the mouse: Multifocal axonopathy, neuroinflammation, and neurodegeneration in the visual system. Exp Neurol, 275 Pt 3, 436-449.

Yang, Z., Wang, P., Morgan, D. et al. (2015) Temporal MRI characterization, neurobiochemical and neurobehavioral changes in a mouse repetitive concussive head injury model. Sci Rep, 5, 11178.

Yates, N. J., Lydiard, S., Fehily, B., Weir, G., Chin, A., Bartlett, C. A., Alderson, J. and Fitzgerald, M. (2017) Repeated mild traumatic brain injury in female rats increases lipid peroxidation in neurons. Exp Brain Res, 235, 2133-2149.

This article is protected by copyright. All rights reserved. 
Zhang, L., Palmer, R. and McClellan, A. D. (2004) Conditioning lesions enhance axonal regeneration of descending brain neurons in spinal-cord-transected larval lamprey. J Comp Neurol, 478, 395-404.

This article is protected by copyright. All rights reserved. 
Figure 1

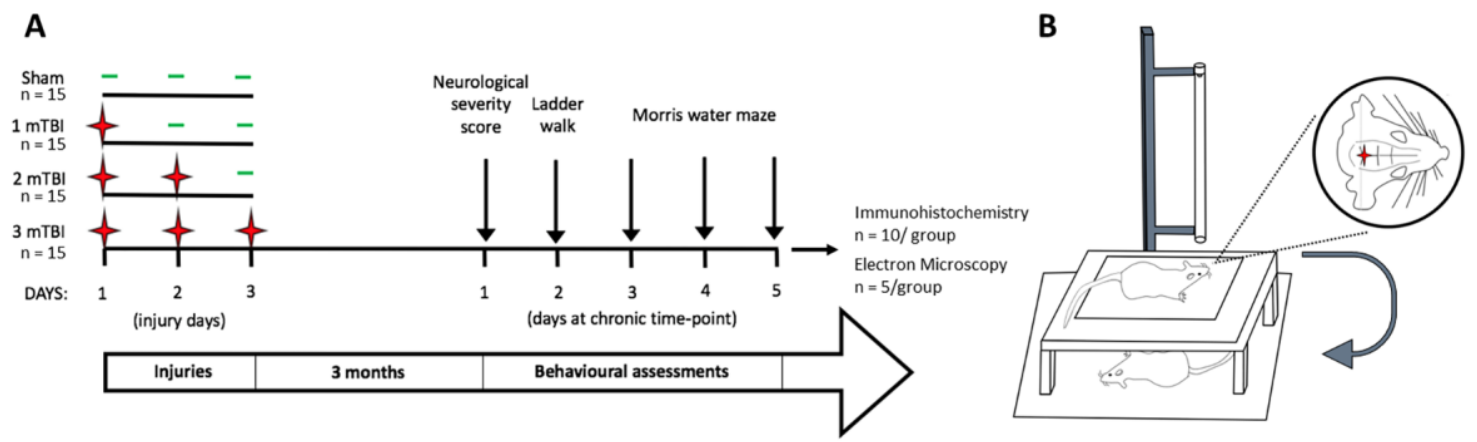

This article is protected by copyright. All rights reserved. 
Figure 2

A
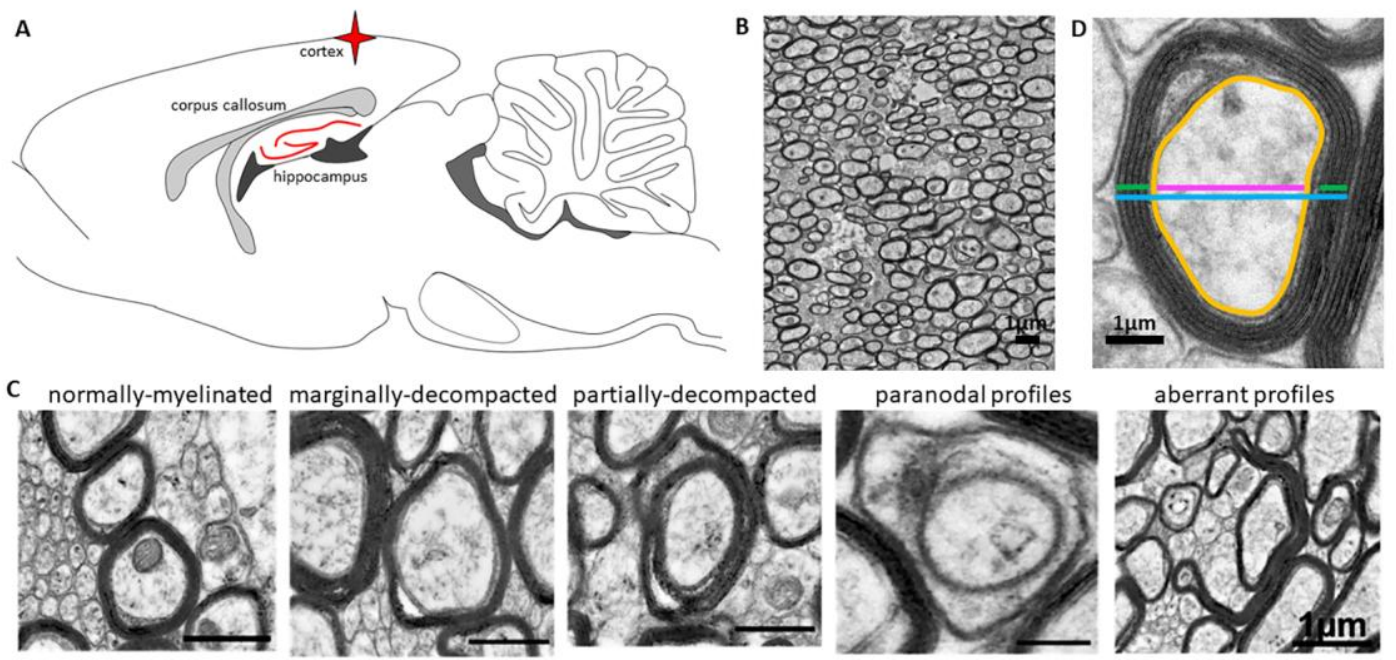

This article is protected by copyright. All rights reserved. 
Figure 3

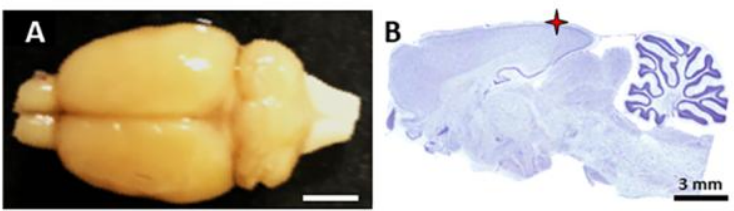

This article is protected by copyright. All rights reserved. 
Figure 4
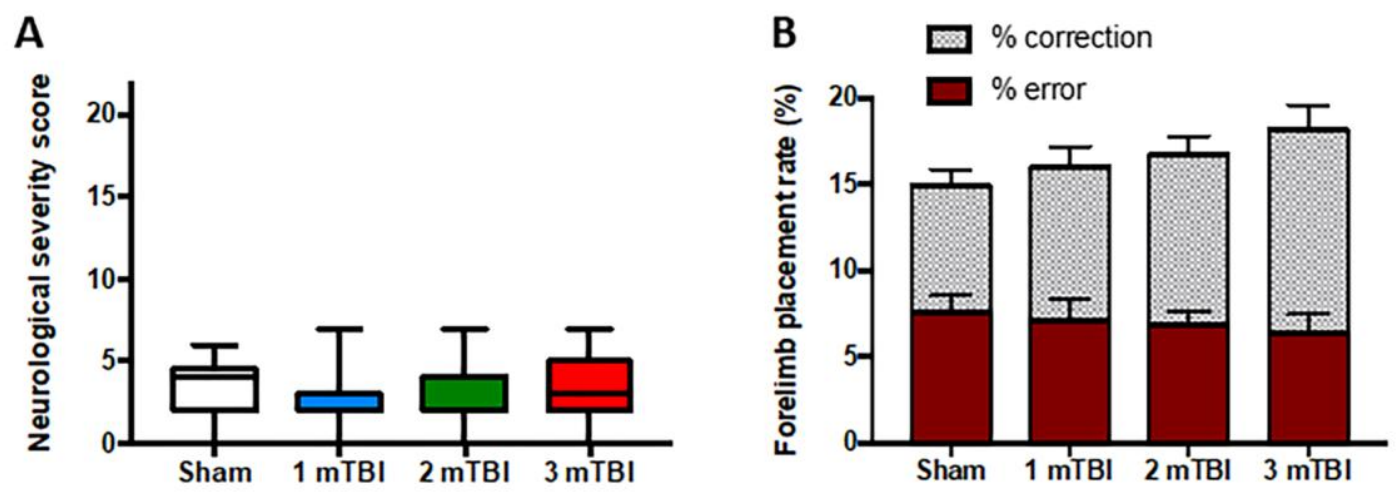

This article is protected by copyright. All rights reserved. 
Figure 5

A
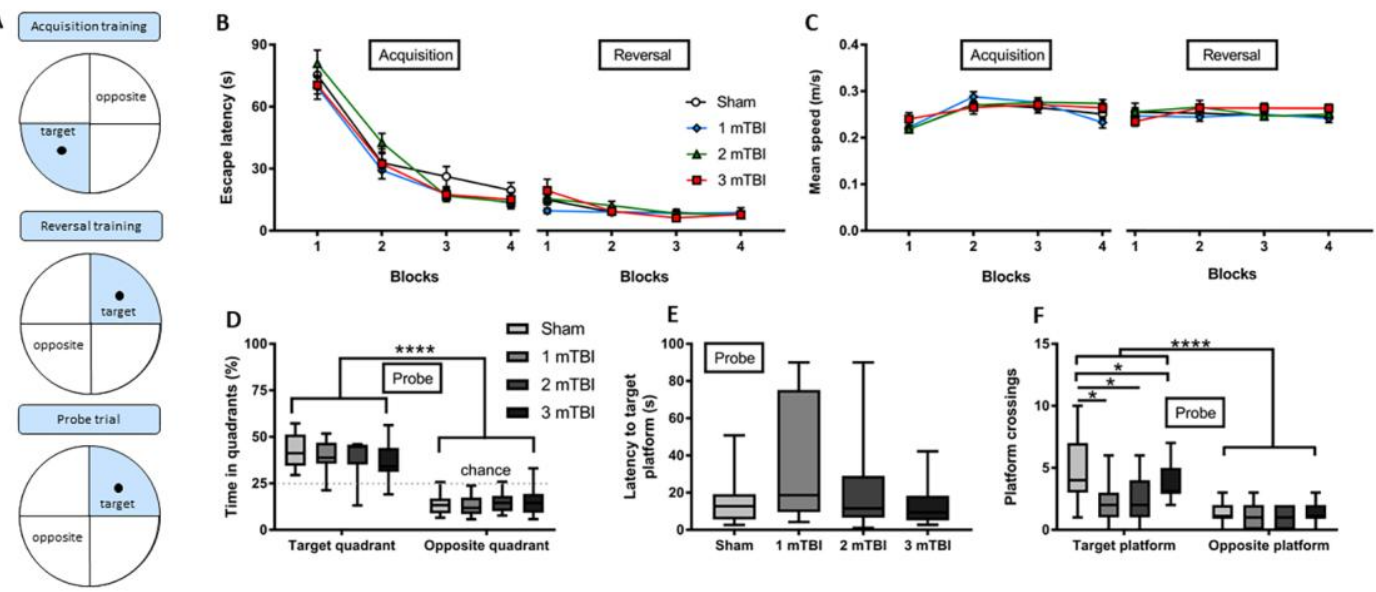

This article is protected by copyright. All rights reserved. 
Figure 6

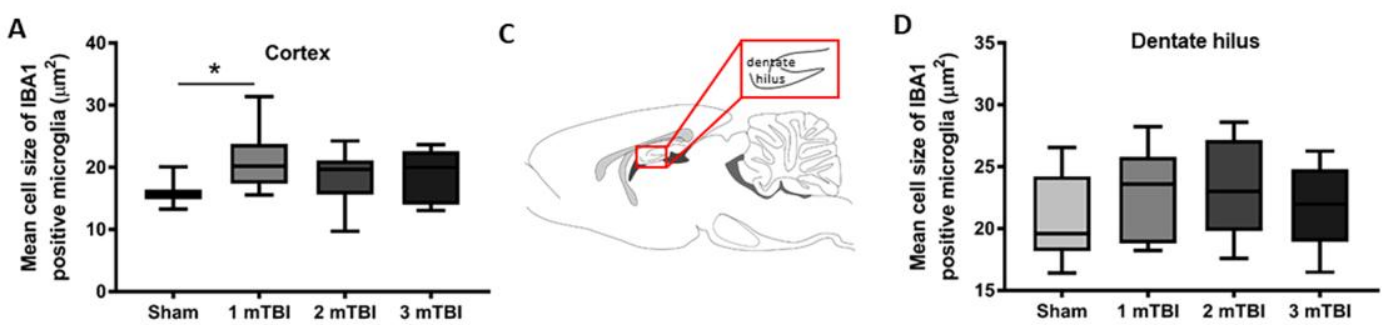

B
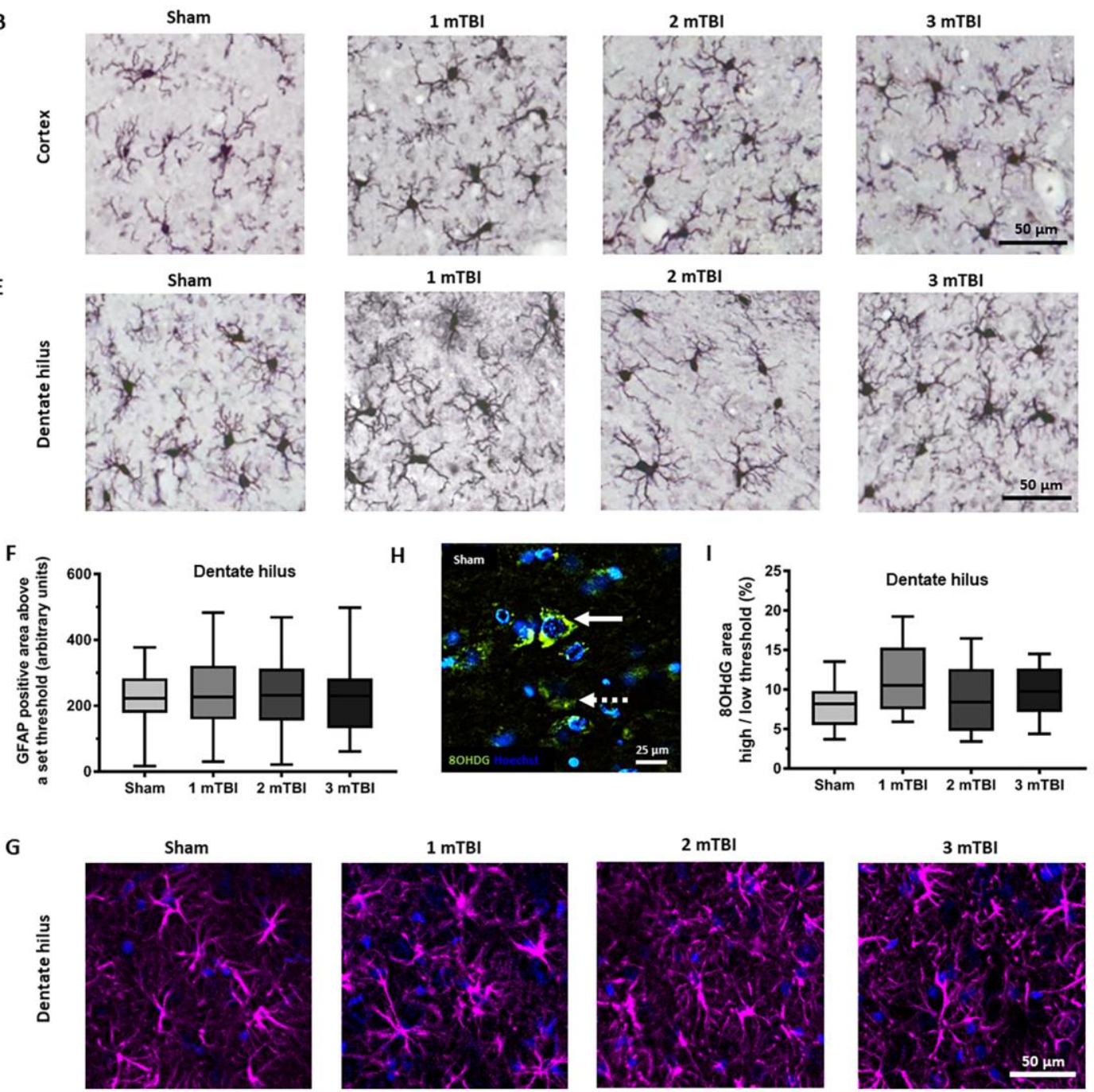

This article is protected by copyright. All rights reserved. 
Figure 7

A

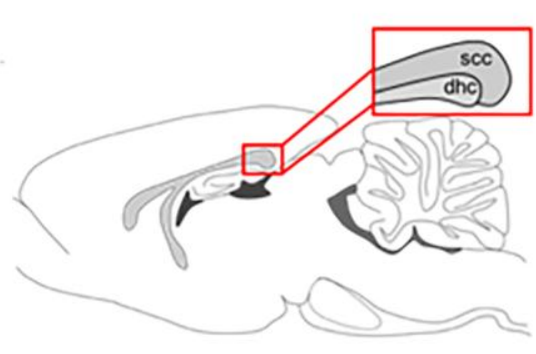

C

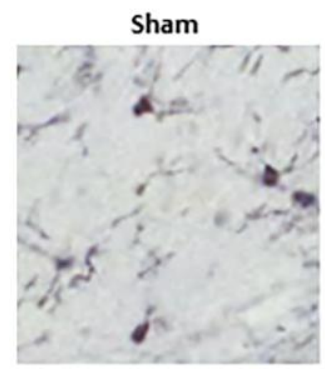

D

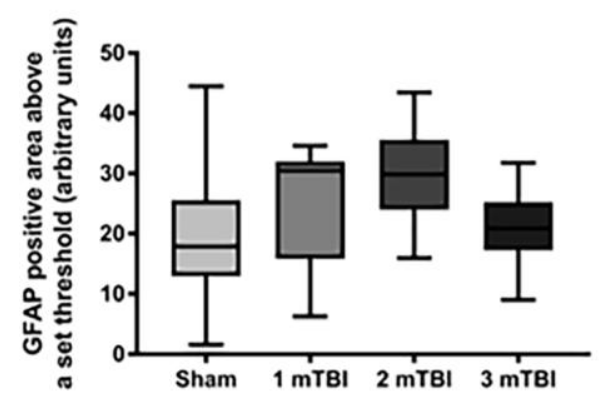

1 mTBI
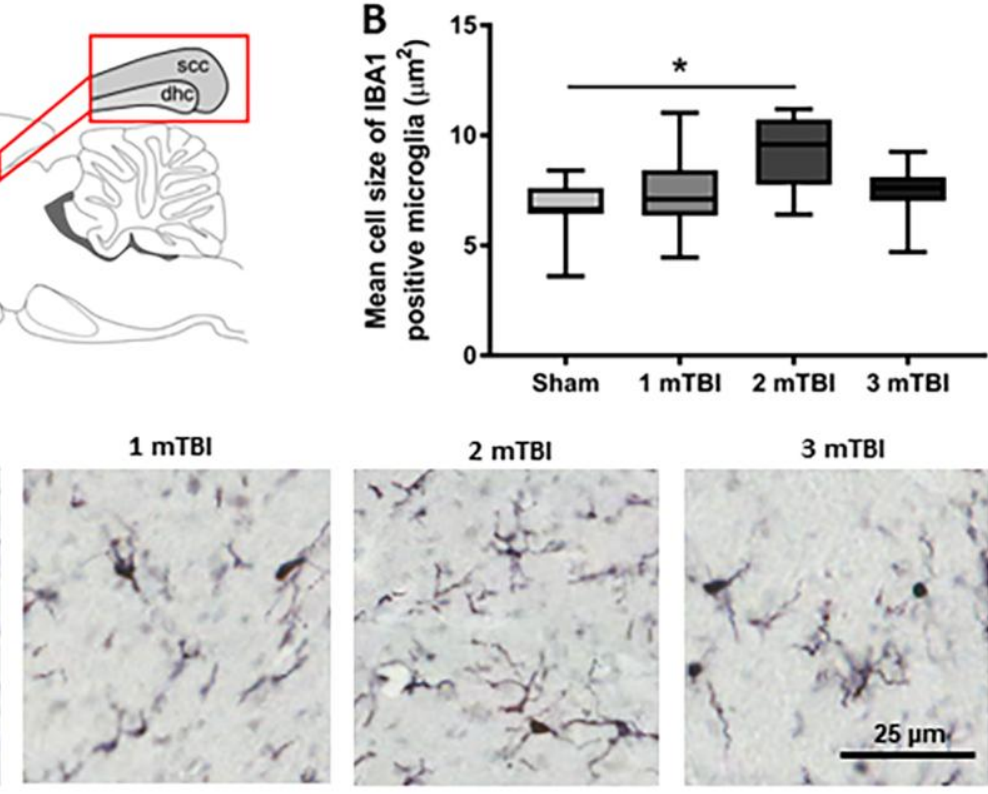

$\mathrm{E}$

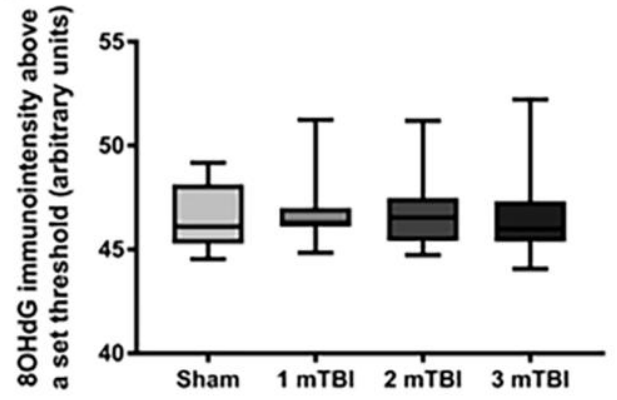

This article is protected by copyright. All rights reserved. 
Figure 8
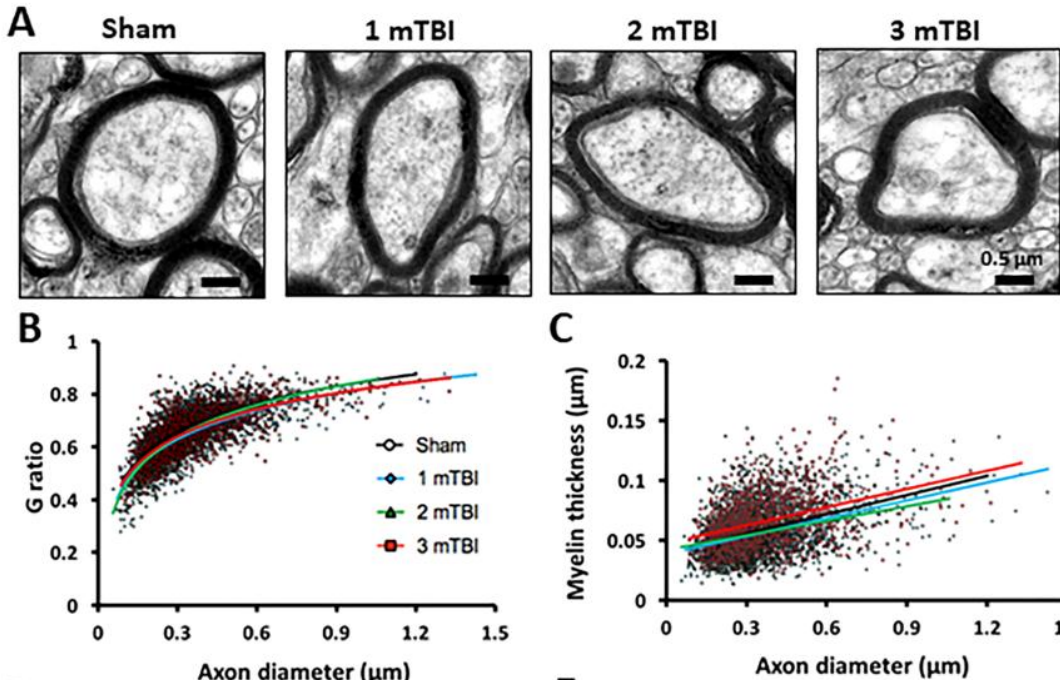

C

D
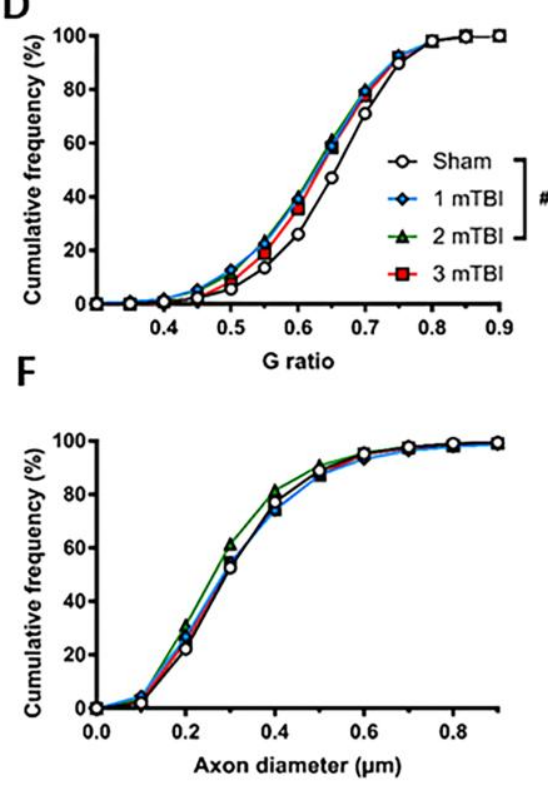

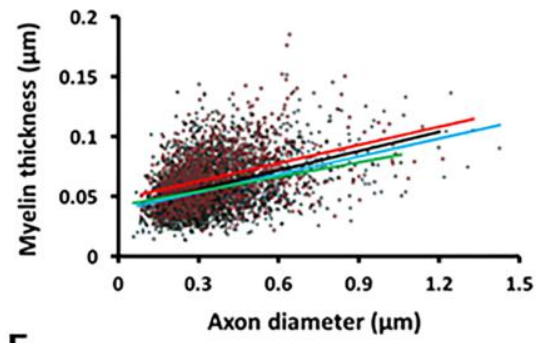

E

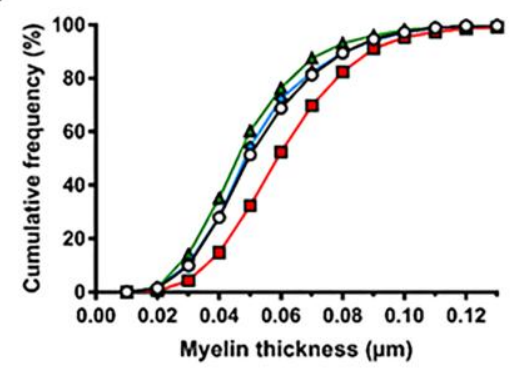

This article is protected by copyright. All rights reserved. 